TRANSACTIONS OF THE

AMERICAN MATHEMATICAL SOCIETY

Volume 357, Number 7, Pages 2799-2827

S 0002-9947(04)03589-5

Article electronically published on September 23, 2004

\title{
POISSON BRACKETS ASSOCIATED TO THE CONFORMAL GEOMETRY OF CURVES
}

\author{
G. MARÍ BEFFA
}

\begin{abstract}
In this paper we present an invariant moving frame, in the group theoretical sense, along curves in the Möbius sphere. This moving frame will describe the relationship between all conformal differential invariants for curves that appear in the literature. Using this frame we first show that the KacMoody Poisson bracket on $\operatorname{Lo}(n+1,1)^{*}$ can be Poisson reduced to the space of conformal differential invariants of curves. The resulting bracket will be the conformal analogue of the Adler-Gel'fand-Dikii bracket. Secondly, a conformally invariant flow of curves induces naturally an evolution on the differential invariants of the flow. We give the conditions on the invariant flow ensuring that the induced evolution is Hamiltonian with respect to the reduced Poisson bracket. Because of a certain parallelism with the Euclidean case we study what we call Frenet and natural cases. We comment on the implications for completely integrable systems, and describe conformal analogues of the Hasimoto transformation.
\end{abstract}

\section{INTRODUCTION}

The theory of completely integrable partial differential equations and the theory of infinite dimensional Poisson manifolds are very closely linked. In fact, the integration of the majority of completely integrable PDEs relies on the existence of two compatible Hamiltonian structures with respect to which the system is Hamiltonian (that is, the system is bi-Hamiltonian.) If one of the Hamiltonian structures is nondegenerate and can be inverted, a recursion operator can be defined, generating a family of integrals or preserved quantities of the flow, effectively integrating the system.

The particular case of completely integrable systems linked to the finite dimensional differential geometry of curves has been studied in depth recently. Hasimoto ( $\mathrm{Ha}$ ) showed that the equation for curvature and torsion of the Vortex filament flow was linked geometrically to the nonlinear Shrödinger equation, a well-known integrable system. More developments in that direction were obtained later in [MW], [LP1], [LP2], DSa, [YS] and [MSW], among them a theorem stating that the Hasimoto transformation is a map taking curvature and torsion to the so-called natural curvatures of a curve in Euclidean $\mathbb{R}^{3}$. A rich family of Poisson brackets was produced in the case of a manifold with constant curvature. The Hasimoto map is also a Poisson map, that is, it takes Hamiltonian structures for the NLS

Received by the editors June 11, 2003 and, in revised form, November 14, 2003.

2000 Mathematics Subject Classification. Primary 37K25, 37K05, 37K10; Secondary 53A55.

Key words and phrases. Invariant evolutions of curves, conformal curves, Poisson brackets, differential invariants, completely integrable PDEs. 
equation to Hamiltonian structures for the Vortex filament flow. The Poisson structures themselves can be defined using an invariant Riemannian moving frame along the flow. This point of view allowed the generalization of these Poisson brackets to the $n$-dimensional Euclidean space and the generalization of the Hasimoto transformation as the map taking Frenet curvatures to natural curvatures ([M1]). A vector modified $\mathrm{KdV}$ system of equations was linked to these structures in the $n$-dimensional Euclidean case ([TT], TU]). Again, the general Poisson structures used to integrate these systems can be defined simply with the use of an invariant Frenet moving frame along the flow. More specifically, the Hamiltonian structures are reductions of a more general Poisson structure defined on the space of loops in $o(n)^{*}$ to the submanifold of Frenet coframes or natural coframes ([M1]).

Surprisingly enough, this relation also exists in other kind of geometries. In the projective case, the Adler-Gel'fand-Dikii bracket or second Hamiltonian structure for generalized KdV system of equations is obtained by reducing a Poisson structure defined on the space of loops in $\operatorname{sl}(n+1)^{*}$ to a certain submanifold of matrices containing the projective differential invariants of curves ([DS], [M2]). We will call these matrices the Frenet projective matrices, they are of the form

$$
\left(\begin{array}{ccccc}
0 & k_{1} & k_{2} & \ldots & k_{n} \\
1 & 0 & 0 & \ldots & 0 \\
0 & 1 & 0 & \ldots & 0 \\
\ldots & \ddots & \ddots & \ldots & \ldots \\
0 & 0 & \ldots & 1 & 0
\end{array}\right)
$$

and they can be written as $\rho^{-1} \frac{d}{d x} \rho$, where $\rho$ is a certain projectively invariant moving frame (in the group theoretical sense) along a curve in a manifold with a projective structure (in the theory of differential invariants these are called moving coframes.) In [M2] the author classified all invariant flows of curves in $\mathbb{R} P^{n}$ inducing Hamiltonian evolutions on their differential invariants. There is an intriguing and fundamental difference between the Euclidean and projective cases. When we consider invariant flows in the Euclidean case, these need to be parametrized by arc-length and the invariant flow of curves needs to be arc-length preserving. In the projective case we need to allow the element of arc-length to flow also and hence we must consider arbitrary parametrizations. In the projective arc-length preserving case one does not obtain an associated Hamiltonian structure. The reason for this difference is still unclear but could have a very interesting geometrical interpretation.

In this paper we present the study of the Möbius sphere (the flat conformal case, since it is a local study.) A classification of differential invariants of curves in a manifold with a conformal structure was originally published by Fialkow ( $\mathrm{F}]$ ), and a second one was done by the author in [M3. In Section 3 we use the second classification to produce a group theoretical moving frame $\rho$ along a curve $u$. A moving frame in this sense is not known for a curve in a conformal manifold. We then show that $\rho^{-1} \frac{d}{d x} \rho$ equals what we call the conformal Frenet matrices, whose shape also appears for the case $n=3$ in Sharpe's book ([Sh]), obtained in a completely different way. Thus we describe the exact relationship between all the conformal differential invariants of curves that appear in the literature. We also introduce what we call the conformal natural curvatures, which have a similar role to the natural Euclidean ones, and the associated natural moving frame. 
In Section 4 we introduce the Poisson bracket in the space of loops in $o(n+1,1)^{*}$ and we show that we can reduce it to the space of Frenet matrices. This way we obtain what we call the conformal Adler-Gel'fand-Dikii bracket. We show that the bracket can also be reduced to the space of conformal natural matrices and that the map taking natural curvatures to conformal Frenet curvatures is a Poisson map. This map is the generalization of the Hasimoto transformation to the conformal case.

In Section 5 we classify those conformally invariant flows of curves which induce a Hamiltonian evolution on their conformal differential invariants. We first use the moving frame $\rho$ to write explicitly the evolution induced on the conformal curvatures by the flow. We then describe the reduced bracket and establish the relation between both evolutions. Surprisingly enough one needs to allow the element of arc-length to evolve also, just as in the projective case. So somehow, the conformal case seems to be related to the Euclidean case by the existence of natural curvatures, Hasimoto transformations, etc., but also linked to the projective case by the reduction process which is different from the Euclidean one and closely resembles the projective one. We do both regular and natural cases in parallel even though the natural case can only be done formally, or under different assumptions. But, as it happens in the Euclidean case, the conditions for the flow to produce a Hamiltonian flow on the curvatures are easier to check in the natural case, and hence they are likely to be favoured by someone working in completely integrable systems.

Section 6 presents open problems and describes implications for completely integrable systems.

The author would like to thank the students in her Math 376 Honors class for throwing a party to celebrate the results in this paper. She appreciates their encouragement.

\section{Definitions AND BASIC RESUlts}

In this section we will provide the background definitions and results in Differential Geometry and invariant theory needed throughout the paper. Much of it is stated as in the two excellent books [Sh] and [O].

\subsection{Cartan connections and conformal geometry.}

Definition 1. Let $G$ be a Lie group and let $H \subset G$ be a closed subgroup such that $G / H$ is connected. The pair $(G, H)$ is called a Klein Geometry.

Assume that a group $G$ acts transitively on a manifold $M$, and let $H_{p}=\{g \in$ $G$, such that $g . p=p\}$. The manifold $M=G / H_{p}$ is called a homogeneous space. Examples of homogeneous spaces are Euclidean and projective spaces and the Möbius sphere.

The definition of Klein geometry is due to Klein and Killing. They developed the idea of classical geometries as homogeneous spaces with Klein geometries for which the principal group $G$ preserves the associated geometric axioms which traditionally defined the geometries since Euclid. For example, the $n$-dimensional Euclidean space is a homogeneous space given by $\mathbb{R}^{n}=E u c_{n}(\mathbb{R}) / S O_{n}(\mathbb{R})$. By $E u c_{n}(\mathbb{R})$ we denote the Euclidean group defined as

$$
\operatorname{Euc}_{n}(\mathbb{R})=\left\{\left(\begin{array}{ll}
1 & 0 \\
v & \vartheta
\end{array}\right)\right\}
$$


$\vartheta \in S O_{n}(\mathbb{R})$, where we identify $S O_{n}(\mathbb{R})$ with its copy inside $E u c_{n}(\mathbb{R})$ (that is, $v=0$.) $E u c_{n}(\mathbb{R})$ acts on $\mathbb{R}^{n}$ by multiplication of matrices if we identify $x \in \mathbb{R}^{n}$ with $\left(\begin{array}{l}1 \\ x\end{array}\right)$. The subgroup $S O_{n}(\mathbb{R})$ leaves the origin fixed.

Definition 2. If $P$ is the standard $H_{p}$ principal bundle associated to the homogeneous space $G / H_{p}$, a $\mathfrak{g}$-valued 1 -form on $P, \omega$, is called a Cartan connection if it satisfies the following conditions:

(i) for each point $p \in P$, the linear map $\omega_{p}: T_{p} P \rightarrow \mathfrak{g}$ is an isomorphism;

(ii) $\left(R_{h}\right)^{*} \omega=\operatorname{Ad}\left(h^{-1}\right) \omega$ for all $h \in H$;

(iii) $\omega(0, X)=X$ for all $X \in \mathfrak{h}$

where, as usual, $R_{h}$ denotes the right multiplication map, $h \in H, A d$ represents the Adjoint action of the group, and $(0, X)$ is a trivialization of the element in $P$ associated to $X \in \mathfrak{h}$.

In the case of a flat homogenous space, the Cartan connection is locally given by the pullback via a Cartan gauge (a choice of a local section in $G / H$ ) of the MaurerCartan form in $G$. This pullback immediately satisfies the structural equation

$$
d \omega+\frac{1}{2}[\omega, \omega]=0 .
$$

See $\underline{\text { Sh }}$ for more details.

Consider the Lorentz space $\mathbb{R}^{n+2}$ with the indefinite quadratic form defined by the matrix

$$
J=\left(\begin{array}{ccc}
0 & 0 & -1 \\
0 & I & 0 \\
-1 & 0 & 0
\end{array}\right),
$$

where $I$ is the identity matrix. Let $L$ be the light cone, that is, the submanifold of vectors $v$ such that

$$
v^{T} J v=0 .
$$

The projectivisation of $L$ is called the Möbius sphere, and we will denote it by $S^{n}$. The group $O(n+1,1)$ of matrices preserving $J$,

$$
O(n+1,1)=\left\{R \in G L(n+2) ; \quad R^{T} J R=J\right\}
$$

acts on $S^{n}$ in the following manner: clearly $O(n+1,1)$ preserves $L$, since it preserves $J$. An element in $u \in S^{n}$ can be identified with an element of $L$ given by the lift $(q, u, 1) \in L$ with $-2 q+\sum_{k=1}^{n}\left(u^{k}\right)^{2}=0$, a property that is necessary to belong to the light cone. The action of $O(n+1,1)$ on the sphere $S^{n}$ is given by the projectivisation of the action of an element of $O(n+1,1)$ on $(q, u, 1)$. For more details see $\mathrm{Sh}$ or $\mathrm{GS}$.

The group $P O(n+1,1)=O(n+1,1) / \pm I$ is called the Möbius group. Let $H$ be the subgroup of $P O(n+1,1)$ leaving $e_{1}^{n+2}$ invariant (here $e_{1}^{n+2}$ is the canonical vector $e_{1}$ in $\left.\mathbb{R}^{n+2}\right)$. It is easy to see that $H$ is the projection on $P O(n+1,1)$ of the subgroup of $O(n+1,1)$ defined by matrices of the form

$$
\left(\begin{array}{ccc}
\epsilon & 0 & 0 \\
0 & \Theta & 0 \\
0 & 0 & -\epsilon
\end{array}\right)\left(\begin{array}{ccc}
1 & \xi^{T} & 1 / 2 \xi^{T} \xi \\
0 & 1 & \xi \\
0 & 0 & 1
\end{array}\right)
$$

with $\epsilon>0$ and $\Theta \in O(n)$. 
Definition 3. The pair $(P O(n+1,1), H)$ is called the Möbius model.

It is known that $P O(n+1,1)$ acts on $S^{n}$ as a group of conformal transformations. It is also known that the Möbius sphere is diffeomorphic to the flat homogeneous space $P O(n+1,1) / H$. For more information see [Sh], [GS] or [K].

2.2. Moving frames. Let $M$ be an $n$-dimensional homogeneous space with $G$ a Lie group acting transitively on $M$. Let $J^{(m)}=J^{(m)}(\mathbb{R}, M)$ denote the $m$ th order jet bundle consisting of equivalence classes of parametrized curves modulo $m$ th order contact. We introduce local coordinates $x$ on $\mathbb{R}$, and $u=\left(u^{\alpha}\right)$ on $M$. The induced local coordinates on $J^{(m)}$ are denoted by $u_{k}$, with components $u_{k}^{\alpha}$, where $u_{k}^{\alpha}=\frac{d^{k} u^{\alpha}}{d x^{k}}, 0 \leq k \leq m, \alpha=1, \ldots, n$, represent the derivatives of the dependent variables $-u^{\alpha}-$ with respect to the independent variable $-x-$.

Since $G$ preserves the order of contact between submanifolds, there is an induced action of $G$ on the jet bundle $J^{(m)}$ known as its $m$ th prolongation, and denoted by $G^{(m)}$ (the underlying group being identical to $G$ ). The group $G$ does not act on $x$, and the prolonged action becomes quite simple, namely the action is given by

$$
\begin{gathered}
G^{(m)} \times J^{(m)} \rightarrow J^{(m)}, \\
\left(g, u_{k}\right) \rightarrow(g \cdot u)_{k} .
\end{gathered}
$$

Recall that, if a group $G$ acts on a manifold $M$, then there is an induced action on the space of vector fields on $M$ given by a multiplier representation with a Jacobian multiplier (or weight, see [O] for more information). Hence, the following definition of invariant vector field is very natural.

Definition 4. A map (or tangent vector) $F: J^{(m)} \rightarrow \mathbb{R}^{n}$ is called a (infinitesimal) relative vector differential invariant of the action of $G$ with Jacobian weight if for any infinitesimal generator of the group action, $w$, defined as $w=\sum_{i=1}^{n} \xi^{i} \frac{\partial}{\partial u^{i}}, F$ satisfies

$$
\operatorname{pr}^{(m)} w(F)=\frac{\partial \xi}{\partial u} F
$$

where $\frac{\partial \xi}{\partial u}$ is the Jacobian and where $\operatorname{pr}^{(m)} w(F)$ represents the application of the prolonged vector field (infinitesimal generator for the prolonged action) $\mathrm{pr}^{(m)} w$ to each one of the entries in $F$.

A map $I: J^{(m)} \rightarrow \mathbb{R}$ is called a $m$ th order differential invariant if it is invariant under the prolonged action of $G^{(m)}$. From an infinitesimal point of view, $I$ is a differential invariant whenever $\operatorname{pr}^{(m)} w(I)=0$ for any infinitesimal generator $w$.

Definition 5. An $n$th order (left) moving frame is a map $\rho^{(n)}: J^{(n)} \rightarrow G$ which is (locally) $G$-equivariant with respect to the prolonged action of $G$ on $J^{(n)}$, and the left action of $G$ on itself.

The pull-back of the left-invariant Maurer-Cartan form on $G$ by a moving frame $\rho^{(n)}$ is called an $n$th order (left) moving coframe.

It is known (see $[\mathrm{O}]$ ) that a complete set of differential invariants can be found among the coefficients of a moving coframe, that is, when all possible dependencies have been found among the moving coframe entries. In fact, classical Frenet equations are a simple reformulation of this method. For an effective and systematic method applying these ideas, see [FO1] and [FO2]. 


\subsection{Differential invariants and classical moving frames for curves in the} Möbius sphere. The classical method of moving frames is due to Cartan [C] and relies on the identification of an invariant frame (a set of $n$-independent and invariant vectors) along the curve $u$. This frame is usually called the classical moving frame. For example, in the Euclidean case, one looks for an orthonormal frame along the curve, the Frenet frame. From the group theoretical point of view classical invariant frames are formed by what we called above relative invariants with Jacobian weight.

The relationship between classical and group theoretical moving frames is known only in some particular cases. For example, if $F$ has in columns the Frenet frame along a Euclidean curve $u$, the frame $\rho: J^{(n)} \rightarrow \operatorname{Euc}(n)$,

$$
\rho\left(u^{(n)}\right)=\left(\begin{array}{ll}
1 & 0 \\
u & F
\end{array}\right)
$$

where $\operatorname{Euc}(n)$ is the Euclidean group, is a moving frame in the group theoretical sense. Analogous formulations can also be found for the projective case; see Section 7 in [FO1]. In the next section we will find a group theoretical moving frame for a curve in the Möbius sphere and we will establish the proper relation between classical and group theoretical frames. A classical moving frame was found by Fialkow in $[\mathrm{F}]$ and by the author in [M1] using different approaches.

What follows is a description of the classical frame and differential invariants as found in M1.

Let $S$ be the Möbius sphere and let $u: I \rightarrow S$ be a parametrized nondegenerate curve, where $I$ is some interval in $\mathbb{R}$. We denote by $p_{i, j}$ the expression

$$
p_{i, j}=\frac{u_{i} \cdot u_{j}}{u_{1} \cdot u_{1}}
$$

where we are denoting by $\cdot$ the usual dot product in $\mathbb{R}^{n}$. From now on we will denote by $\ell$ the length $\left(u_{1} \cdot u_{1}\right)^{\frac{1}{2}}$ and, along the curve $u$, we will define the inner product $\langle v, w\rangle=\frac{1}{\ell^{2}} v \cdot w$, so that $p_{i, j}=\left\langle u_{i}, u_{j}\right\rangle$. Assume we define $g_{s}^{r}$ via the following recursion fomula

$$
g_{s}^{r+1}=\frac{d}{d x} g_{s}^{r}-p_{1,2} g_{s}^{r}+g_{s-1}^{r},
$$

for $s \geq 2, r \geq 3$ with initial conditions $g_{r}^{r}=1$ for all $r, g_{1}^{2}=-2 p_{1,2}$ by definition, and

$$
g_{1}^{r}=-\sum_{s=2}^{r} p_{i, s} g_{s}^{r} .
$$

The following two theorems describe the invariant frame and differential invariants for parametrized conformal curves.

Theorem 1. The vectors

$$
G_{1}=u_{1}, \quad G_{r}=\sum_{s=1}^{r+1} g_{s}^{r+1} u_{s},
$$

for $r=2, \ldots, n$, form an invariant frame (in the classical sense) along the curve $u$. That is, they are relative invariants with Jacobian weight for the prolonged action of $O(n+1,1)$. 
Notice that, from (2.6) all $G_{r}$ are orthogonal to $G_{1}$. The following two properties of the invariant frame can also be found in [M1].

Proposition 1. Let $\left\{G_{i}\right\}$ be given as in Theorem 1. Then, the following holds true:

- The products $\left\langle G_{i}, G_{j}\right\rangle$ are all differential invariants for the curve $u$.

- The following relation is satisfied for $i=2, \ldots, n-1$

$$
\frac{d}{d x}\left(\frac{G_{i}}{\ell}\right)=\frac{G_{i+1}}{\ell}-G_{1}\left(\frac{G_{i}}{\ell} \cdot u_{2}\right)
$$

The following theorem naturally identifies a set of generators for the differential invariants.

Theorem 2. Let $I_{r}, r=1, \ldots, n$ be defined as

$$
I_{1}=p_{1,3}+\frac{3}{2} p_{2,2}-3 p_{1,2}^{2}, \quad I_{r}=\frac{1}{\ell^{2}} G_{r} \cdot G_{r}
$$

for $r=2, \ldots, n$. Then $\left\{I_{1}, \ldots, I_{n}\right\}$ form a complete set of generating and independent differential invariants for $u$. Furthermore, $I_{2}^{1 / 4}$ is an element of arc-length.

Example 1. In the case $n=2$ we have $g_{3}^{3}=1, g_{2}^{3}=-3 p_{1,2}$ and $g_{1}^{3}=p_{1,2}^{2}-p_{1,3}$ from formula (2.3). Therefore, a classical moving frame will be given by

$$
G_{1}=u_{1}, \quad G_{2}=u_{3}-3 p_{1,2} u_{2}+\left(p_{1,2}^{2}-p_{1,3}\right) u_{1} .
$$

The differential invariants are $I_{1}$ as above and $I_{2}=p_{3,3}-6 p_{1,2} p_{2,3}-p_{1,3}^{2}+6 p_{1,2}^{2} p_{1,3}+$ $9 p_{1,2}^{2} p_{2,2}-5 p_{1,2}^{4}$ as arc-length parameter.

These frames and invariants are relative and absolute invariants, respectively, under the action of the conformal group, but they are not invariant under reparametrizations. In [M1] we described how any orthonormalization of $\left\{G_{i}\right\}$ with respect to the inner product $\langle$,$\rangle would be an invariant frame, invariant also under$ reparametrizations. We chose a simple Gramm-Schmidt process there, but a Frenet process would also work, as shown below. As in the Euclidean case, we need to assume a certain nondegeneracy of the curve $u$.

Theorem 3. Let $\left\{G_{i}\right\}$ be the invariant frame obtained in Theorem 1. Then, for a generic curve, there exists an invariant frame $\left\{F_{1}, F_{2}, \ldots, F_{n}\right\}$ such that $F_{1} \cdot F_{i}=0$, for $i=2, \ldots, n$, and such that if $\widehat{F}$ has $\left\{F_{2}, \ldots, F_{n}\right\}$ in columns, then

$$
\frac{\widehat{F}^{T}}{\ell} \frac{d}{d x}\left(\frac{\widehat{F}}{\ell}\right)=K_{2}
$$

where

$$
K_{2}=\left(\begin{array}{ccccc}
0 & -\kappa_{2} & 0 & \ldots & 0 \\
\kappa_{2} & 0 & -\kappa_{3} & \ldots & 0 \\
\vdots & \ddots & \ddots & \ddots & \vdots \\
0 & \ldots & \kappa_{n-2} & 0 & -\kappa_{n-1} \\
0 & \ldots & 0 & \kappa_{n-1} & 0
\end{array}\right) .
$$

The functions $\kappa_{1}=-I_{1}$ and $\kappa_{i}, i=2, \ldots, n-1$, together with the arc-length $s=I_{2}^{1 / 4}$ form a generating set of conformal differential invariants for the curve $u$.

These differential invariants coincide with the ones presented in $[\mathrm{F}]$. 
Proof. The construction of $\left\{F_{i}\right\}$ is very similar to that of a Frenet frame in Euclidean geometry. The main difference is that $F_{1}=G_{1}$ needs to remain orthogonal to the rest of the frame, and so we need to project on $F_{1}^{\perp}$ after each differentiation. That is, the $G_{1}$ term in (2.7) needs to be systematically ignored along the construction. This is, of course, necessary to maintain the invariance of the frame.

The first 2 vectors of the frame are straightforward. Let $F_{1}=G_{1}$ and $F_{2}=\frac{G_{2}}{I_{2}^{1 / 2}}$ so that $\left\langle F_{2}, F_{2}\right\rangle=1$. Now, consider the vector $\widehat{F_{2}^{\prime}}$ representing the projection on $F_{1}^{\perp}$ of the vector $\ell \frac{d}{d x}\left(\frac{F_{2}}{\ell}\right)$. That is, using Proposition 1 we have

$$
\widehat{F_{2}^{\prime}}=\frac{d}{d x}\left(\frac{1}{I_{2}^{1 / 2}}\right) G_{2}+\frac{1}{I_{2}^{1 / 2}} G_{3} .
$$

Clearly, since $\left\langle F_{2}, F_{2}\right\rangle=1, \widehat{F_{2}^{\prime}}$ is orthogonal to both $F_{1}$ and $F_{2}$. Assume that

$$
\left\langle\widehat{F_{2}^{\prime}}, \widehat{F_{2}^{\prime}}\right\rangle=\frac{I_{3}}{I_{2}}-\frac{3}{4} \frac{\left(I_{2}^{\prime}\right)^{2}}{I_{2}^{2}}>0
$$

and let $\kappa_{2}^{2}=\left\langle\widehat{F_{2}^{\prime}}, \widehat{F_{2}^{\prime}}\right\rangle$. We call

$$
F_{3}=\frac{1}{\kappa_{2}} \widehat{F_{2}^{\prime}} .
$$

Clearly these three vectors are invariant vectors since they are invariant combinations of $G_{i}$. Again, consider the vector $\widehat{F_{3}^{\prime}}+\kappa_{2} F_{2}$, where $\widehat{F_{3}^{\prime}}$ represents the projection on $F_{1}^{\perp}$ of the vector $\ell \frac{d}{d x}\left(\frac{F_{3}}{\ell}\right)$. That is, $\widehat{F_{3}^{\prime}}=\frac{d}{d x}\left(\frac{1}{\kappa_{2}}\right) G_{3}+\left(\frac{1}{\kappa_{2}}\right) G_{4}$. As in the Euclidean case, $\widehat{F_{3}^{\prime}}+\kappa_{2} F_{2}$ is orthogonal to $F_{i}, i=1,2,3$. Assume

$$
\kappa_{3}^{2}=\left\langle\widehat{F_{3}^{\prime}}+\kappa_{2} F_{2}, \widehat{F_{3}^{\prime}}+\kappa_{2} F_{2}\right\rangle>0 \text {. }
$$

Then we denote

$$
F_{4}=\frac{1}{\kappa_{3}}\left(\widehat{F_{3}^{\prime}}+\kappa_{2} F_{2}\right) .
$$

Again, the vector $F_{4}$ is invariant since it is an invariant combination of the frame $\left\{G_{i}\right\}$. We repeat the procedure with the vector $\widehat{F_{4}^{\prime}}+\kappa_{3} F_{3}$ and the process goes on until we complete a set of $n$ vectors and $n-2$ invariants $\kappa_{j}, j=2, \ldots, n-1$. It is clear that, together with $I_{1}$ and $I_{2}$, the invariants $\kappa_{j}$ form a generating set of invariants, since the $I_{j}$ invariants are generators and the $\kappa_{j}$ are formed from the invariants $I_{j}, j=1, \ldots, n$ increasing the differentiation order by one at each step. The need to insert a minus sign on $I_{1}$ to define $\kappa_{1}$ will become clear in the next section.

The Frenet procedure is not the only one to determine an orthonormal frame along a curve. In $[\mathrm{B}]$, the author explained a different approach and the definition of the so-called natural frame in Euclidean geometry. The same procedure can be applied here in the production of $\left\{F_{2}, \ldots, F_{n}\right\}$, resulting on what one can call the conformal natural frame. For more details in the Euclidean case, please see $[B]$.

Theorem 4. For a generic curve, there exists an invariant frame $\left\{V_{1}, V_{2}, \ldots, V_{n}\right\}$ such that $V_{1} \cdot V_{i}=0$, for $i=2, \ldots, n$, and such that if $\widehat{V}$ has $\left\{V_{2}, \ldots, V_{n}\right\}$ in columns, then

$$
\frac{\widehat{V}^{T}}{\ell} \frac{d}{d x}\left(\frac{\widehat{V}}{\ell}\right)=N_{2}
$$


where

$$
N_{2}=\left(\begin{array}{cccc}
0 & -\nu_{2} & \ldots & -\nu_{n-1} \\
\nu_{2} & 0 & \ldots & 0 \\
\vdots & \vdots & \vdots & \vdots \\
\nu_{n-1} & 0 & \ldots & 0
\end{array}\right)
$$

The functions $\nu_{1}=-I_{1}$ and $\nu_{i}, i=2, \ldots, n-1$, together with the arc-length $s=I_{2}^{1 / 4}$ form a generating set of conformal differential invariants for the curve $u$, which we will call natural curvatures. They are unique up to the action of $O(n-2)$.

Proof. Let $\left\{F_{i}\right\}$ be the invariant frame obtained in Theorem 3 We will show that there exists a matrix function of the form $\left(\begin{array}{ll}1 & 0 \\ 0 & \theta\end{array}\right)$ with $\theta(x) \in O(n-2)$ such that

$$
\hat{V}=\hat{F}\left(\begin{array}{ll}
1 & 0 \\
0 & \theta
\end{array}\right)
$$

satisfies equation (2.10). Since $\left\{V_{1}, V_{2}, \ldots, V_{n}\right\}$ will also be an orthonormalization with respect to $\langle$,$\rangle of \left\{G_{1}, \ldots, G_{n}\right\}$ as in Theorem 11 they are also an invariant classical frame. Therefore, $\nu$ as in the theorem will be a generating system of differential invariants for the curve.

Indeed, substituting in (2.10) we obtain

$$
\frac{\widehat{V}^{T}}{\ell} \frac{d}{d x}\left(\frac{\widehat{V}}{\ell}\right)=\left(\begin{array}{cc}
1 & 0 \\
0 & \theta^{T}
\end{array}\right)\left(\begin{array}{cc}
0 & 0 \\
0 & \theta^{\prime}
\end{array}\right)+\left(\begin{array}{cc}
1 & 0 \\
0 & \theta^{T}
\end{array}\right) K_{2}\left(\begin{array}{cc}
1 & 0 \\
0 & \theta
\end{array}\right) .
$$

If we write $K_{2}=\left(\begin{array}{cc}0 & -\kappa_{2} e_{1}^{n-1} \\ \kappa_{2} e_{1}^{n-1} & K_{3}\end{array}\right)$, where $e_{1}^{n-1}$ is the canonical vector $e_{1}$ in $\mathbb{R}^{n-1}$, we can rewrite the right-hand side above as

$$
\left(\begin{array}{cc}
0 & -\kappa_{2} e_{1}^{n-1} \theta \\
\kappa_{2} \Theta^{T} e_{1}^{n-1} & \theta^{T} \theta^{\prime}+\theta^{T} K_{3} \theta
\end{array}\right) .
$$

Therefore, if we impose condition (2.10) we have

$$
\nu=-\kappa_{2} \theta^{T} e_{1}^{n-1}, \quad \theta^{\prime}=-K_{3} \theta .
$$

This determines $\nu$ from $\kappa$ up to a constant matrix in $O(n-2)$. Also, if $\nu$ is known, $\kappa_{2}=\|\nu\|$ and we can construct $K_{3}$ using the first row of $\Theta$ (that is, $-\frac{1}{\kappa_{2}} \nu$ ) to initiate a process similar to the construction of the Euclidean Frenet frame.

\section{MOVInG FRAMES FOR CONFORMAL CURVES}

In this section we will describe a moving frame along a curve on the Möbius sphere in the group theoretical sense. That is, this time we will find an equivariant map

$$
\rho: J^{(k)} \rightarrow O(n+1,1) .
$$

We will use this map to describe the evolution of the conformal differential invariants of a curve induced by an invariant curve evolution of the type (3.9) below and also to describe a Poisson reduction procedure which will result in the conformal Adler-Gelfand-Dikii bracket. This will be carried out in the next section. 
Theorem 5. Let $u: \mathbb{R} \rightarrow S$ be a curve on the Möbius sphere.

Let $F_{K}$ be the matrix containing the invariant vectors $F_{i}, i=1, \ldots, n$, in columns, and let $F_{i}$ be as in Theorem $\mathbf{3}$.

Let $\mathbf{q}_{K}$ be the vector given by $\mathbf{q}_{K}=\left(q_{i}\right)$ where $q_{i}=\left\langle F_{i}, u_{2}\right\rangle$, whenever $i=$ $2, \ldots, n$ and $q_{1}=-\left\langle F_{1}, u_{2}\right\rangle$ by definition.

Then, the map $\rho_{K}: J^{(n+1)} \rightarrow O(n+1,1)$ is defined as

$$
\rho_{K}\left(u^{(n+1)}\right)=\left(\begin{array}{ccc}
1 & u^{T} & \frac{1}{2} u \cdot u \\
0 & I & u \\
0 & 0 & 1
\end{array}\right)\left(\begin{array}{ccc}
\ell & 0 & 0 \\
\frac{1}{\ell} F_{K} \mathbf{q}_{K} & \frac{1}{\ell} F_{K} & \\
\frac{1}{2 \ell} \mathbf{q}_{K} \cdot \mathbf{q}_{K} & \frac{1}{\ell} \mathbf{q}_{K}^{T} & \frac{1}{\ell}
\end{array}\right) .
$$

The result also holds true if we substitute the Frenet frame $\left\{F_{i}\right\}$ by the natural frame $\left\{V_{i}\right\}$.

Proof. One can prove directly that, if $\rho_{K}$ is given as in (3.1), then

$$
\rho_{K}^{T} J \rho_{K}=J
$$

where $J$ is as in (2.2). That is, $\rho_{K}\left(u^{(n+1)}\right) \in O(n+1,1)$ (in fact, the above is a standard factorization of elements in $O(n+1,1)$.) This map is equivariant since it is a solution of the equation (3.2) below, where $K$ is a matrix whose entries are all differential invariants of the action (either the Frenet conformal curvatures or the natural ones, depending on the choice of frame). Notice that in the proof of the next theorem we only make use of $\rho_{K}\left(u^{(n+1)}\right) \in O(n+1,1)$.

Theorem 6. Let $\rho_{K}$ be the frame in (3.1). Then

$$
\hat{K}=\rho_{K}^{-1} \frac{d}{d x} \rho_{K}=\left(\begin{array}{ccc}
0 & e_{1}^{T} & 0 \\
\kappa_{1} e_{1}+s^{2} e_{2} & K & e_{1} \\
0 & \kappa_{1} e_{1}^{T}+s^{2} e_{2}^{T} & 0
\end{array}\right)
$$

where $s$ is the element of arc-length $\left(s^{4}=I_{2}\right)$ and where $K$ is given by

$$
K=\left(\begin{array}{cc}
0 & 0 \\
0 & K_{2}
\end{array}\right)
$$

$K_{2}$ as in (2.9), if $\rho_{K}$ is defined using the Frenet frame, or

$$
K=\left(\begin{array}{cc}
0 & 0 \\
0 & N_{2}
\end{array}\right)
$$

$N_{2}$ as in (2.11), if $\rho_{K}$ is defined using the natural frame.

Notice that the shape of $\hat{K}$ given as in (3.2) appears in [Sh]. Even though the approach is different here (they use group theoretical reduction rather than explicitly finding moving frames), the theorem above establishes a direct correspondence between all conformal differential invariants of curves that appear in the literature.

Proof. First of all, since $\rho_{K}\left(u^{(k)}\right) \in O(n+1,1)$ we have that $\rho_{K}^{-1}=J \rho_{K}^{T} J$, where $J$ is given as in (2.2). That is,

$$
\rho_{K}=\left(\begin{array}{ccc}
a_{K} & \mathbf{v}_{K}^{T} & b_{K} \\
P_{K}^{0} & P_{K} & P_{K}^{n+1} \\
c_{K} & \mathbf{w}_{K}^{T} & d_{K}
\end{array}\right), \quad \rho_{K}^{-1}=\left(\begin{array}{ccc}
d_{K} & -\left(P_{K}^{n+1}\right)^{T} & b_{K} \\
-\mathbf{w}_{K} & P_{K}^{T} & -\mathbf{v}_{K} \\
c_{K} & -\left(P_{K}^{0}\right)^{T} & a_{K}
\end{array}\right)
$$


where $P_{K}=\frac{1}{\ell}\left(F_{K}+u \mathbf{q}_{K}^{T}\right), P_{K}^{0}=\frac{1}{\ell}\left(u_{2}-2 p_{1,2} u_{1}+\frac{1}{2} p_{2,2} u\right), P_{K}^{n+1}=\frac{u}{\ell}$ and where $a_{K}, b_{K}, c_{K}, d_{K}$ are given by

$$
\begin{aligned}
& d_{K}=\frac{1}{\ell}, \quad b_{K}=\frac{1}{2 \ell} u \cdot u, \quad c_{K}=\frac{1}{2 \ell} p_{2,2}, \\
& a_{K}=\ell\left(1+p_{2,0}-2 p_{1,2} p_{1,0}+\frac{1}{4} p_{0,0} p_{2,2}\right),
\end{aligned}
$$

and

$$
\mathbf{v}_{K}=\frac{1}{\ell} F_{K}^{T} u+\frac{\ell}{2} \mathbf{q}_{K} p_{0,0}, \quad \mathbf{w}_{K}=\frac{1}{\ell} \mathbf{q}_{K} .
$$

From here

$$
\rho_{K}^{-1} \frac{d}{d x} \rho_{K}
$$

$$
=\left(\begin{array}{ccc}
-c_{K} b_{K}^{\prime}+\left(P_{K}^{0}\right)^{T}\left(P_{K}^{n+1}\right)^{\prime}-a_{K} d_{K}^{\prime} & \left(-\mathbf{w}_{K} b_{K}^{\prime}+P_{K}^{T}\left(P_{K}^{n+1}\right)^{\prime}-\mathbf{v}_{K} d_{K}^{\prime}\right)^{T} & 0 \\
-\mathbf{w}_{K} a_{K}^{\prime}+P_{K}^{T}\left(P_{K}^{0}\right)^{\prime}-\mathbf{v}_{K} c_{K}^{\prime} & -\mathbf{w}_{K}\left(\mathbf{v}_{K}^{T}\right)^{\prime}+P_{K}^{T} P k^{\prime}-\mathbf{v}_{K}\left(\mathbf{w}_{K}^{T}\right)^{\prime} & * \\
0 & * & *
\end{array}\right),
$$

where $*$ represents entries which are determined by the ones shown and the fact that $\rho_{K}^{-1} \frac{d}{d x} \rho_{K}$ is in the algebra $o(n+1,1)$. (For convenience, the tilde represents differentiation with respect to $x$.)

We will analyze the entries one by one. Indeed, straightforward calculations using definitions in Theorem [5] show that

$$
c_{K} b_{K}^{\prime}-\left(P_{K}^{0}\right)^{T}\left(P_{K}^{n+1}\right)^{\prime}+a_{K} d_{K}^{\prime}=0 .
$$

Using $p_{i, j}^{\prime}=p_{i+1, j}+p_{i, j+1}-2 p_{1,2}$ we immediately obtain

$$
-\mathbf{w}_{K} b_{K}^{\prime}+P_{K}^{T}\left(P_{K}^{n+1}\right)^{\prime}-\mathbf{v}_{K} d_{K}^{\prime}=\frac{1}{\ell^{2}} F_{K}^{T} u_{1}=e_{1} .
$$

Also direct computations give us

$$
\begin{gathered}
\mathbf{w}_{K} a_{K}^{\prime}+P_{K}^{T}\left(P_{K}^{0}\right)^{\prime}-\mathbf{v}_{K} c_{K}^{\prime} \\
=\frac{1}{\ell^{2}} F_{K}^{T}\left(u_{3}-3 p_{1,2} u_{2}+\left(-p_{1,3}+3 p_{1,2}^{2}\right) u_{1}\right)+\left(-p_{1,3}+\frac{3}{2} p_{2,2}+3 p_{1,2}^{2}\right) e_{1} .
\end{gathered}
$$

Clearly, the coefficient of $e_{1}$ equals $-I_{1}=\kappa_{1}$ as in Theorem 3 and the factor companion of $F_{K}^{T}$ is the vector $G_{2}=I_{2}^{1 / 2} F_{2}$ as described in Theorem 3 also. Given that $F$ contains in columns an orthonormal system with respect to $\langle$,$\rangle , and given$ that $I_{2}^{1 / 4}=s$ is the element of arc-length, we have that $F_{K}^{T} G_{2}=s^{2} e_{2} \ell^{2}$ and so we get

$$
-\mathbf{w}_{K} a_{K}^{\prime}+P_{K}^{T}\left(P_{K}^{0}\right)^{\prime}-\mathbf{v}_{K} c_{K}^{\prime}=\kappa_{1} e_{1}+s^{2} e_{2} .
$$

Finally we need to study the central $o(n)$ block of (3.8). This can be rewritten as

$$
-\mathbf{w}_{K}\left(\mathbf{v}_{K}^{T}\right)^{\prime}+P_{K}^{T} P_{K}^{\prime}-\mathbf{v}_{K}\left(\mathbf{w}_{K}^{T}\right)^{\prime}=\frac{F_{K}^{T}}{\ell}\left(\frac{F_{K}}{\ell}\right)^{\prime}+\frac{1}{\ell^{2}}\left(F_{K}^{T} u_{1} \mathbf{q}_{K}^{T}-\mathbf{q}_{K} u_{1}^{T} F_{K}\right) .
$$

It is straightforward to check that the first row and column of $\frac{F_{K}^{T}}{\ell}\left(\frac{F_{K}}{\ell}\right)^{\prime}$ are given by $\frac{1}{\ell^{2}}\left(\mathbf{q}_{K} e_{1}^{T}-e_{1} \mathbf{q}_{K}^{T}\right)$. Application of Theorem 3 or Theorem 4, depending on whether we use Frenet or natural frames, concludes the proof.

The last theorem involving moving frames will be very useful to us in the next section. 
Theorem 7. Assume a curve $u$ is evolving following an invariant evolution of the form

$$
u_{t}=F \mathbf{r}
$$

where $F$ is the matrix containing in columns an invariant frame (either Frenet or natural) and where $\mathbf{r}$ is an invariant vector, that is, a vector depending on the differential invariants and their derivatives with respect to the parameter $x$. Let $\rho_{K}$ be the moving frame along $u$ given in (3.1) and assume $\frac{d}{d t} \rho_{K}$ is the evolution induced by (3.9) on $\rho_{K}$. Then

$$
\rho_{K}^{-1} \frac{d}{d t} \rho_{K}=\left(\begin{array}{ccc}
\epsilon & \mathbf{r}^{T} & 0 \\
\xi & A & \mathbf{r} \\
0 & \xi^{T} & -\epsilon
\end{array}\right) .
$$

The entries $\epsilon, \xi, A$ in the matrix (3.10) can be easily obtained through direct algebraic manipulations as we will see in the next section.

Proof. The proof of this theorem is a simple calculation. Using formula (3.5) for $\rho_{K}^{-1}$, the entries of $\rho_{K}^{-1} \frac{d}{d t} \rho_{K}$ in place $(1, i)$ with $i=2, \ldots, n+1$ are given by the vector

$$
-\mathbf{w}_{K}\left(b_{K}\right)_{t}+P_{K}^{T}\left(P_{K}^{n+1}\right)_{t}-\mathbf{v}\left(d_{K}\right)_{t}
$$

where the subindex indicates total differentiation with respect to $t$. If we expand the expression we obtain

$$
-\frac{1}{2 \ell} \mathbf{q}_{K}\left(\frac{u \cdot u}{\ell}\right)_{t}+\frac{1}{\ell} F_{K}^{T}\left(\frac{u}{\ell}\right)_{t}+\frac{1}{\ell} \mathbf{q}_{K} u^{T}\left(\frac{u}{\ell}\right)_{t}-\frac{1}{\ell} F_{K}^{T} u\left(\frac{1}{\ell}\right)_{t}-\frac{1}{2} \ell \mathbf{q}_{K} p_{0,0}\left(\frac{1}{\ell}\right)_{t}
$$

which equals

$$
\frac{1}{\ell^{2}} F_{K}^{T} u_{t}=\frac{1}{\ell^{2}} F_{K}^{T} F_{K} \mathbf{r}=\mathbf{r}
$$

\section{Poisson REduction to the Submanifold OF CONFORMAL DIFFERENTIAL INVARIANTS}

The initial content of this section will seem to be unrelated to the previous comments and results. The connection between these two apparently unrelated subjects will become clear through the reduction process in the second subsection.

First we will describe the Poisson manifold we will be working on, its Poisson bracket, and its relation to moving frames.

4.1. The Lie-Poisson bracket on duals of Kac-Moody algebras. Let $G$ be a semisimple Lie group, and $\mathfrak{g}$ its Lie algebra. Let $L G=C^{\infty}\left(S^{1}, G\right)$ be the group of loops on $G$ and let $L \mathfrak{g}=C^{\infty}\left(S^{1}, \mathfrak{g}\right)$ be its Lie algebra. Let $L \mathfrak{g}^{*}=C^{\infty}\left(S^{1}, \mathfrak{g}^{*}\right)$ be its dual (it is not really its dual but what is called the regular part of the dual, dense in the dual of the algebra of loops). The space of loops could be replaced by functions from $\mathbb{R}$ to $G$ vanishing at infinity, or any condition that ensures that no boundary terms will appear when we integrate by parts. We will do so in the case of natural curvatures where a reduction in a different space (or a formal reduction) is needed. Some problems with classical Poisson brackets arise in the non-periodic 
case so that the periodic case is favoured if possible. See the discussion on [DS]. Let $Q$ be the Killing form associated to $\mathfrak{g}$. Define the following cocycle of the algebra

$$
w(N, M)=\int_{S^{1}} Q\left(N, \frac{d M}{d x}\right) d x
$$

for any $N, M \in L \mathfrak{g}$. The form $w$ is called a cocycle because it has the properties necessary to guarantee that $L \mathfrak{g} \oplus \mathbb{R}$ is a Lie algebra with Lie bracket given by

$$
[(N, s),(M, r)]=([N, M], w(N, M)) .
$$

This algebra is called the central extension of $L \mathfrak{g}$, also known as a Kac-Moody algebra on the circle associated to $\mathfrak{g}$ (we will denote it by $k a c(\mathfrak{g})$.)

The Poisson bracket we are interested in is the Lie-Poisson bracket on the dual of the Kac-Moody algebra. Let's have a quick look at how this bracket looks explicitly. Recall that a pairing between $L \mathfrak{g}$ and $L \mathfrak{g}^{*}$ is given by the integral of $\frac{1}{2}$ times the trace, as it is used below. Recall also that the pairing between the central extension algebra and its dual is given by

$$
\langle(L, s),(C, t)\rangle=\int_{S^{1}} \operatorname{tr}(L C) d x+s t
$$

where $\operatorname{tr}$ denotes $\frac{1}{2}$ times the trace. In the case at hand, if we have a functional $G: L \mathfrak{g}^{*} \rightarrow \mathbb{R}$, its variational derivative, $\frac{\delta G}{\delta L}$, is given by an element of $L \mathfrak{g}$ and it is defined by the Frechet derivative of the functional, namely

$$
\left.\frac{d}{d \epsilon}\right|_{\epsilon=0} G(L+\epsilon V)=\int_{S^{1}} \operatorname{tr}\left(\frac{\delta G}{\delta L} V\right) d x .
$$

Finally, it is known ([Ki] that the coadjoint action of the Kac-Moody group on $k a c^{*}(\mathfrak{g})$ reduces to the following action of the group of loops

$$
A(g)(L, s)=\left(-s g^{-1} g^{\prime}+g^{-1} L g, s\right) .
$$

It is customary to identify an element $(L, s) \in k a c^{*}(\mathfrak{g})$ with the differential operator

$$
-s \frac{d}{d x}+L
$$

so that the action above corresponds to conjugation of such operator by $g \in L G$. This conjugation (or gauge) by $g$ corresponds to the change of variable $X g=Y$ on the solutions of the system $-s X^{\prime}=X L$.

With these descriptions in mind, the Lie-Poisson bracket on $L \mathfrak{g}^{*}$ is defined as

$$
\begin{gathered}
\{H, G\}(L, s)=\left\langle\left[\left(\frac{\delta H}{\delta L}, \frac{\delta H}{\delta s}\right),\left(\frac{\delta G}{\delta L}, \frac{\delta G}{\delta s}\right)\right],(L, s)\right\rangle=\left\langle\left[\frac{\delta H}{\delta L}, \frac{\delta G}{\delta L}\right], L\right\rangle+s w\left(\frac{\delta H}{\delta L}, \frac{\delta G}{\delta L}\right) \\
=\int_{S^{1}} \operatorname{tr}\left(\frac{\delta G}{\delta L}\left(-s\left(\frac{\delta H}{\delta L}\right)^{\prime}+\left[L, \frac{\delta H}{\delta L}\right]\right)\right) d x .
\end{gathered}
$$

From this expression we readily see that the Hamiltonian vector field associated to the Hamiltonian $H$ is given by

$$
\xi_{H}=-s\left(\frac{\delta H}{\delta L}\right)^{\prime}+\left[L, \frac{\delta H}{\delta L}\right]
$$

Notice that the variational derivative of the functionals $H$ and $G$ in the central component $s$ do not appear in the formula of the Poisson bracket. Thus, the dual of the Kac-Moody algebra foliates into affine Poisson submanifolds, each one 
corresponding to a fixed value of $s$. In particular we will work in the Poisson submanifold corresponding to the choice $s=-1$.

4.2. The Poisson bracket on the space of conformal differential invariants: The conformal Adler-Gel'fand-Dikii bracket. In this section we aim to show that the general Lie-Poisson bracket defined on the dual of a Kac-Moody algebra associated to $O(n+1,1)$ can be successfully Poisson reduced to the submanifold of matrices of the form (3.2), with $K$ having either the Frenet or the natural form. In the natural case we will need to assume conditions other than periodicity, for example entries vanishing at infinity or a formal reduction, and we will need to quotient by $O(n-2)$. The resulting Poisson bracket, especially in the Frenet case, will be a conformal analogue of the so-called Adler-Gel'fand-Dikii bracket, which is associated to the projective geometry of curves. We will later use the construction in this section to identify which curve evolutions induce a Hamiltonian evolution on its invariants $\kappa=\left(\kappa_{j}\right)$ or $\nu=\left(\nu_{i}\right)$. This result is of special interest in the study of completely integrable systems. We will comment on the consequences for that subject in the last section.

Let $\rho$ be a fundamental matrix solution of the differential equation

$$
\rho^{\prime}=\rho M
$$

where $M \in L o(n+1,1)$ is of the form

$$
M=\left(\begin{array}{ccc}
-\epsilon & \beta e_{1}^{T} & 0 \\
\alpha & C & \beta e_{1} \\
0 & \alpha^{T} & \epsilon
\end{array}\right)
$$

with $C \in \operatorname{Lo}(n)$ and where $\epsilon, \alpha$ and $\beta$ are also $C^{\infty}$ and periodic, $\beta>0$. First, by standard Floquet theory, $\rho$ has a monodromy, that is, there exists $\Gamma \in O(n+1,1)$ such that $\rho(x+2 \pi)=\Gamma \rho(x)$ for any $x$. Assume

$$
\rho=\left(\begin{array}{ccc}
a & \mathbf{v}^{T} & b \\
P^{0} & P & P^{n+1} \\
c & \mathbf{w} & d
\end{array}\right) .
$$

Assume further that $d$ never vanishes. Define

$$
u=\frac{P^{n+1}}{d}, \quad \mathbf{q}=\frac{\mathbf{w}}{d}, \quad F=\frac{1}{d} P-u \mathbf{q}^{T} .
$$

Theorem 8. The rest of the entries in $\rho$ are determined by $d, u, \mathbf{q}$ and $F$. In fact,

$$
\rho=\left(\begin{array}{ccc}
1 & u^{T} & \frac{1}{2} u \cdot u \\
0 & I & u \\
0 & 0 & 1
\end{array}\right)\left(\begin{array}{ccc}
\frac{1}{d} & 0 & 0 \\
d F \mathbf{q} & d F & 0 \\
\frac{d}{2} \mathbf{q} \cdot \mathbf{q} & d \mathbf{q}^{T} & d
\end{array}\right) .
$$

One also has the additional relations

$$
F^{T} F=\frac{1}{d^{2}} I, \quad d^{2} F^{T} u_{1}=\beta e_{1} .
$$

Proof. The proof is straightforward since the above is a standard factorization of elements in $O(n+1,1)$. Condition

$$
\rho^{-1} \rho^{\prime}=M,
$$

in particular the fact that $M$ has $e_{1}$ in its expression, implies $d^{2} F^{T} u_{1}=\beta e_{1}$. 
We will denote $\rho$ as in (4.6) by $\rho(d, u, \mathbf{q}, F)$. Clearly, the conformal moving frame described in Theorem 5 equals $\rho_{K}=\rho\left(d_{K}, u, \mathbf{q}_{K}, F_{K}\right)$, where $d_{K}, u, \mathbf{q}_{K}, F_{K}$ are given in that same theorem.

Notice that, since $F$ has in columns an orthogonal basis with respect to the dot product, condition $d^{2} F^{T} u_{1}=\beta e_{1}$ implies that $F_{1}$ is a multiple of $u_{1}$. If we further use $F^{T} F=\frac{1}{d^{2}} I$, we have that

$$
F_{1}=\frac{1}{d\left(u_{1} \cdot u_{1}\right)^{1 / 2}} u_{1}
$$

and therefore

$$
\beta=d\left(u_{1} \cdot u_{1}\right)^{1 / 2} .
$$

Now we define the subgroup that will be acting on the manifold $\mathcal{M}$ of loops of the form (4.5).

Theorem 9. Let $\mathcal{H}$ be the subgroup on $L O(n+1,1)$ given by matrices of the form

$$
\left(\begin{array}{ccc}
\delta^{-1} & 0 & 0 \\
\delta^{-1} \Theta \xi & \Theta & 0 \\
\frac{\delta^{-1}}{2} \xi^{T} \xi & \xi^{T} & \delta
\end{array}\right)
$$

where $\Theta=\left(\begin{array}{ll}1 & 0 \\ 0 & \theta\end{array}\right), \theta \in L O(n-1)$ and $\delta>0$. If $g \in \mathcal{H}$, then,

$$
\rho(d, u, \mathbf{q}, F) g=\rho\left(\delta d, u, \delta^{-1}\left(\Theta^{T} \mathbf{q}+\xi\right), \delta^{-1} F \Theta\right) .
$$

Proof. The theorem follows from the fact that

$$
\left(\begin{array}{ccc}
d^{-1} & 0 & 0 \\
d F \mathbf{q} & d F & 0 \\
\frac{d}{2} \mathbf{q} \cdot \mathbf{q} & d \mathbf{q}^{T} & d
\end{array}\right)\left(\begin{array}{ccc}
\delta^{-1} & 0 & 0 \\
\delta^{-1} \Theta \xi & \Theta & 0 \\
\frac{\delta^{-1}}{2} \xi \cdot \xi & \xi^{T} & \delta
\end{array}\right)=\left(\begin{array}{ccc}
(d \delta)^{-1} & 0 & 0 \\
* & d F \Theta & 0 \\
* & d\left(\mathbf{q}^{T} \Theta+\xi^{T}\right) & d \delta
\end{array}\right)
$$

where $*$ represent entries which are determined by the matrix belonging to the group.

We now have the conditions to define the conformal analogue of the projective Adler-Gelfand-Dikii bracket, that is, we are ready to reduce the Kac-Moody Lie Poisson bracket on $\operatorname{kac}^{*}(o(n+1,1))$ to the submanifold of Frenet conformal matrices or the submanifold of natural matrices.

The general reduction procedure that we will apply is identical to the one presented in [MR]: Let $(\mathcal{P},\{\}$,$) be a Poisson manifold, Let \mathcal{M} \subset \mathcal{P}$ be a submanifold and $i: \mathcal{M} \rightarrow \mathcal{P}$ the inclusion. Let $\left.E \subset T \mathcal{P}\right|_{\mathcal{M}}$ be a subbundle of the tangent to $\mathcal{P}$ restricted to $\mathcal{M}$. Assume the following conditions are satisfied:

(a) $E \cap T \mathcal{M}$ is integrable and define a foliaton on $\mathcal{M}$. We will call the foliation $\Phi$.

(b) The space of leaves is a manifold and $\pi: \mathcal{M} \rightarrow \mathcal{M} / \Phi$ is a submersion.

(c) $E$ leaves $\{$,$\} invariant. That is, if \mathcal{F}, \mathcal{G}: \mathcal{P} \rightarrow \mathbb{R}$ are smooth functions on $\mathcal{P}$ with differentials vanishing on $E$, then the differential of $\{\mathcal{F}, \mathcal{G}\}$ also vanish on $E$. One says that $E$ preserves the Poisson bracket $\{$,$\} .$

Definition 6. We say $(\mathcal{P}, \mathcal{M}, E,\{\}$,$) is Poisson reducible if \mathcal{M} / \Phi$ has a Poisson structure, $\{,\}_{R}$ such that for any smooth $f, g: \mathcal{M} / \Phi \rightarrow \mathbb{R}$ and for any smooth extensions $\mathcal{F}, \mathcal{G}$ of $f \circ \pi$ and $g \circ \pi$ with differentials vanishing on $E$ we have

$$
\{\mathcal{F}, \mathcal{G}\} \circ i=\{f, g\}_{R} \circ \pi .
$$


The following theorem can be found in [MR for the finite dimensional case, although it can be applied to infinite dimensional cases, as the authors state. In either case, the geometric picture is very clear.

Theorem 10 (Reduction Theorem). Assume $(\mathcal{P}, \mathcal{M}, E,\{\}$,$) satisfy assumptions$ (a), (b), (c) and let $B: T^{*} \mathcal{P} \rightarrow T \mathcal{P}$ be the map associating to each variational derivative its Hamiltonian vector field. Then, $(\mathcal{P}, \mathcal{M}, E,\{\}$,$) is Poisson reducible$ if, and only if

$$
B\left(E^{0}\right) \subset T \mathcal{M}+E .
$$

Let $\mathcal{M}$ be the subset of $\operatorname{Lo}(n+1,1)^{*}$ given by elements of the form (4.5). Let $E$ be the distribution generated by the Hamiltonian vectors fields corresponding to functionals $\mathcal{F}$ such that $\frac{\delta \mathcal{F}}{\delta M}(M) \in \mathfrak{h}$, for any $M \in \mathcal{M}$, where $\mathfrak{h}$ is the Lie algebra associated to the group $\mathcal{H}$ given as in (4.12). It is known that $E$ is integrable and its leaves coincide with the coadjoint orbits of $\mathcal{H}$ on $\mathcal{M}$. Consider the space of leaves $\mathcal{M} / \mathcal{H}$. The distribution $E$ of Hamiltonian vector fields whose variational derivatives belong to $\mathfrak{h}$ certainly preserve the bracket. Indeed, if $\mathcal{F}$ and $\mathcal{G}$ are such that their variational derivatives belong to $E^{0}$, one can easily check that their Hamiltonian vector fields belong to $\mathfrak{h}^{0}$ along $\mathcal{M}$. That means that, if $\mathcal{R}$ is a functional with $\frac{\delta \mathcal{R}}{\delta M}(M) \in \mathfrak{h}$, then $\{\mathcal{F}, \mathcal{R}\}(M)=\{\mathcal{G}, \mathcal{R}\}(M)=0$ for any $M \in \mathcal{M}$. This implies that the variational derivative of $\{\mathcal{F}, \mathcal{R}\}(M)$ and $\{\mathcal{G}, \mathcal{R}\}(M)$ vanishes on $T \mathcal{M}$, that is,

$$
\frac{\delta\{\mathcal{F}, \mathcal{R}\}}{\delta M}, \frac{\delta\{\mathcal{G}, \mathcal{R}\}}{\delta M} \in T \mathcal{M}^{0}
$$

along $\mathcal{M}$. Jacobi's identity and the fact that $T_{M} \mathcal{M}^{0} \subset \mathfrak{h}$, result on

$$
\{\{\mathcal{G}, \mathcal{F}\}, \mathcal{R}\}(M)=0
$$

for any $M \in \mathcal{M}$.

Theorem 11. The submanifold $\mathcal{K}$ given by the set of all Frenet matrices of the form (3.2) with $\kappa_{i} \in C^{\infty}\left(S^{1}, \mathbb{R}\right), \kappa_{i}>0$ for any $i=2, \ldots, n-1$, is a section transverse to the coadjoint leaves of an open set $U \subset \mathcal{M}$ under the coadjoint action of $\mathcal{H}$. Furthermore, $\left(\operatorname{Lo}(n+1,1)^{*}, \mathcal{M}, E,\{\},\right)$ is Poisson reducible and, hence, there exists a Poisson bracket $\{,\}_{R}$ on $\mathcal{K}$.

Proof. First we will show that the set of leaves intersecting $\mathcal{K}$ is an open set of $L o(n+1,1)^{*}$, let's call it $U$, and that $\mathcal{K}$ can be identified with $U / \mathcal{H}$. We will then show that the reduction theorem applies to our special case.

Let $\Omega$ be a $\mathcal{H}$-coadjoint orbit intersecting $\mathcal{K}$. This means that, for any $M \in \mathcal{M}$, there exists $g \in \mathcal{H}$ such that if $\rho$ is a solution of

$$
\rho^{-1} \rho^{\prime}=M,
$$

then $\rho g$ is a solution of

$$
(\rho g)^{-1}(\rho g)^{\prime}=K \in \mathcal{K} .
$$

In particular, since $d_{K}=\frac{1}{\ell}>0$ and $\delta>0$ in $\mathcal{H}$, the $d$-entry for frames along the orbit never vanish. Now, let $\hat{\Omega}$ be a nearby orbit to $\Omega$ and let $\hat{M} \in \hat{\Omega}$. Because of the continuous dependence of solutions of differential equations, if $\rho(d, u, \mathbf{q}, F)$ is a solution of $\rho^{-1} \rho^{\prime}=\hat{M}$, then $d>0$ also. Now, given $u$ (the curve that $\rho$ depends 
on), we fix $\delta=\frac{1}{d \ell}$ where $\ell=\left(u_{1} \cdot u_{1}\right)^{1 / 2}$. The action of an element of $\mathcal{H}$ is given in Theorem 9 . This choice of $\delta$ accomplishes two things: first $\delta d=\frac{1}{\ell}=d_{K}$, and also

$$
\delta^{2} d^{2} F^{T} u_{1}=\frac{1}{\ell^{2}} F^{T} u_{1}=\delta d \ell e_{1}=e_{1} .
$$

This implies that $\beta$ in (4.11) is 1 and therefore $F_{1}=u_{1}$.

We then fix $\Theta=\left(\begin{array}{ll}1 & 0 \\ 0 & \theta\end{array}\right)$ so that $\delta^{-1} F \Theta=F_{K}$, where $F_{K}$ is the Frenet frame associated to $u$. We further fix $\xi$ so that $\delta^{-1}\left(\Theta^{T} \mathbf{q}+\xi\right)=\mathbf{q}_{K}$. The elements $d_{K}, F_{K}$ and $\mathbf{q}_{K}$ were all defined in Theorem 5 with $K$ being the matrix of conformal differential invariants associated to the curve $u$ given by the frame $\rho$. If $g$ is given as in (4.12) with these choices of $\delta, \Theta$ and $\xi$, we have

$$
\rho(d, u, \mathbf{q}, F) g=\rho\left(\delta d, u, \delta^{-1}\left(\Theta^{T} \mathbf{q}+\xi\right), \delta^{-1} F \Theta\right)=\rho\left(d_{K}, u, \mathbf{q}_{K}, F_{K}\right)=\rho_{K}
$$

and so

$$
g^{-1} g^{\prime}+g^{-1} M g=\hat{K}
$$

We next need to show that $\hat{K}$ has periodic entries, so that we can conclude that $\hat{K} \in \mathcal{K}$ and so we can identify $U / \mathcal{H}$ with $\mathcal{K}$, where $U$ is the open set of all orbits intersecting $\mathcal{K}$.

If $M$ is a periodic matrix, $\rho(d, u, \mathbf{q}, F)$ has a monodromy, that is, there exists $\Gamma \in O(n+1,1)$ such that

$$
\rho(x+T)=\Gamma \rho(x)
$$

for all $x$, where $T$ is the period. Clearly

$$
\rho_{K}(x+T)=\rho(x+T) g=\Gamma \rho(x) g=\Gamma \rho_{K}
$$

so that both have the same monodromy. Hence $\hat{K}$ has also periodic entries.

Finally, we need to apply the Poisson reduction theorem to our case. If we identify $U / \Phi$ with $\mathcal{K}$, the projection is given by taking $M$ to $\hat{K}$ through the map (4.16). From our discussion above, conditions (a)-(c) are satisfied. A short inspection shows that $E^{0}$ coincides with elements $H$ such that $B(H)=\xi_{H} \in \mathfrak{h}^{0}$. Therefore

$$
B\left(E^{0}\right) \subset \mathfrak{h}^{0} \subset T \mathcal{M}
$$

and so application of the reduction theorem completes the proof.

We have a similar theorem in the natural case, but here the reduction is purely formal (or we consider our manifold to be matrices with entries vanishing at infinity rather than periodic ones) and the quotient is given by $\mathcal{K} / O(n-2)$, with $\mathcal{K}$ as in Theorem 6] $K$ as in (3.4).

Theorem 12. The submanifold $\mathcal{K} / O(n-2)$ given by the set of all Frenet matrices of the form (3.2) with $K$ as in (3.4) is a section transverse to the coadjoint leaves of $\mathcal{M}$ under the coadjoint action of $\mathcal{H}$. Furthermore, the triplet $\left(\operatorname{Lo}(n+1,1)^{*}, \mathcal{M},\{\}, E,\right)$ is formally Poisson reducible and, hence, there exists a formal Poisson bracket $\{,\}_{R N}$ on $\mathcal{K} / O(n-2)$.

Proof. The proof is identical to the proof of the previous theorem, once we substitute the frame $F$ by the natural frame. The reduction and the resulting brackets are formal because natural curvatures of conformal frames with a monodromy do not need to be periodic. This is a direct consequence of their definition. 


\section{INVARIANT FLOWS INDUCING A HAMILTONIAN EVOLUTION ON THEIR CONFORMAL DIFFERENTIAL INVARIANTS}

In this section we will write explicitly the condition that a curve evolution of the form (3.9) must hold in order to induce a Hamiltonian evolution on the curvatures with respect to the reduced bracket. Notice that (3.9) is the most general form of an invariant evolution of conformal curves. That is, the most general evolution for which the group leaves the space of solutions invariant. We will analyze both the Frenet and the natural cases. As in the Euclidean case, the condition for the natural curvatures is easier to check than the one for the Frenet curvatures. Thus, from the point of view of integrable systems, they will be a prefered choice. On the other hand, from the mathematical point of view, only the Frenet curvatures offer a reduction in the periodic case.

5.1. Some Euclidean results. Before stating the two main theorems we will recall some results from the Euclidean case that we will need here. The following results and definitions can be found in [M1. From now on, let $s o(n)=\sum_{i=1}^{n-1} \mathfrak{g}_{i} \oplus \mathfrak{g}_{-i}$ be the decomposition of $s o(n)$ given by the usual gradation of $g l(n)$. That is, $\mathfrak{g}_{i}$ has zero entries everywhere except for those in place $(r, r+i)$ for any $r$.

Lemma 1. Let $B$ be in $L o(n)^{*}$ and assume that its first column is fixed to be $\left(\begin{array}{l}0 \\ \mathbf{f}\end{array}\right)$, for some $\mathbf{f}(x) \in \mathbb{R}^{n-1}$. Let $C$ be of the form

$$
C=\left(\begin{array}{cc}
0 & \mathbf{h}^{T} \\
\mathbf{h} & 0
\end{array}\right)
$$

for some $\mathbf{h}(x) \in \mathbb{R}^{n-1}$. Then, for any $R(x) \in \mathfrak{g}_{1} \oplus \mathfrak{g}_{-1}$, condition

$$
B^{\prime}+[R, B]+\varkappa C \in \mathfrak{g}_{1} \oplus \mathfrak{g}_{-1}
$$

completely determines $B$ in terms of $\mathbf{f}$ and $R$ only. We denote $B$ as $B(f)$. Since $B(f)^{\prime}+[R, B(f)]+\varkappa C \in \mathfrak{g}_{1} \oplus \mathfrak{g}_{-1}$, the vector $n-1$ containing the $\mathfrak{g}_{-1}$ components of $B(f)^{\prime}+[R, B(f)]+\varkappa C$ is denoted by $\mathcal{B}(f)+\varkappa \mathcal{C}(h)$, where $\mathcal{B}$ and $\mathcal{C}$ are differential operators.

Lemma 2. Let $A \in L o(n)^{*}$ and assume that the $\mathfrak{g}_{1}$ component of $A$ is fixed, that $i s, A_{i, i+1}=f_{i}, i=1, \ldots, n-1$. Then, for any $R \in \mathfrak{g}_{1} \oplus \mathfrak{g}_{-1}$ condition

$$
A^{\prime}+[R, A]=\left(\begin{array}{cc}
0 & \mathbf{a}^{T} \\
\mathbf{a} & 0
\end{array}\right)
$$

where $\mathbf{a}(x) \in \mathbb{R}^{n-1}$, completely determines $A$. We denote $A$ by $A(f)$, and $\mathbf{a}=\mathcal{A}(f)$, where $\mathcal{A}$ is a differential operator.

One more operator is relevant to our calculations. Assume $A(f)$ is defined as in Lemma 2 above. The matrix $A(f)$ can be written as

$$
A(f)=\left(\begin{array}{cc}
0 & (\mathcal{R} \mathbf{f})^{T} \\
-\mathcal{R} \mathbf{f} & *
\end{array}\right)
$$

for some differential operator $\mathcal{R}$, where $*$ above is not relevant here. The following are some of the known properties about $\mathcal{A}, \mathcal{B}, \mathcal{C}$ and $\mathcal{R}$ that were used in [M1] to define the reduced Euclidean Poisson bracket. 
Proposition 2. If $*$ below denotes the adjoint operator, then:

(1) $\mathcal{B}=-\mathcal{A}^{*}$.

(2) $(\mathcal{C A})^{*}=-\mathcal{C} \mathcal{A}$.

(3) $\mathcal{C}=\mathcal{R}^{*}$.

With the aid of these operators, we can now describe explicitly both the evolution induced on the curvatures by an invariant evolution of the curve, and the reduced Poisson brackets found in the previous sections. We will start with an explicit formula for the evolution induced on the conformal curvatures.

5.2. The invariant evolution of conformal curvatures. We are going to assume that $u$ is a curve on the Möbius sphere. Some results similar to the ones presented here have been obtained (or conjectured) for the case of a Riemannian manifold with constant curvature (see [MSW] and [M1]), but the conformal case (in this case, the Maurer-Cartan form) with constant curvature is still unclear. We will denote by $\omega$ the Cartan connection.

If $\mathbf{v}$ is any vector in $\mathbb{R}^{k}$, for any $k$, abusing the notation, we will denote by $\pi(\mathbf{v})$ the $\mathbb{R}^{k-1}$ vector obtained by projecting $\mathbf{v}$ on its last $k-1$ components. We will not be indicating the value of $k$ in each case so, for example, $\pi^{2}(\mathbf{v})=\pi(\pi(\mathbf{v})) \in \mathbb{R}^{k-2}$ will denote the projection on the last $k-2$ components. Let $\kappa$ denote the vector defined by the Frenet conformal curvatures $\kappa(x)=\left(\kappa_{i}(x)\right) \in \mathbb{R}^{n-1}$, and let $\nu=\left(\nu_{i}\right)$ be the vector defined by the natural conformal curvatures. The following theorems describe the evolution of the curvatures and also how to obtain the remaining entries in $\rho_{K}^{-1} \frac{d}{d t} \rho_{K}$ as in Theorem 7 for both Frenet and natural cases. By $e_{1}^{n-1}$ below we denote the canonical vector $e_{1}$ in $\mathbb{R}^{n-1}$.

Theorem 13. Let $u_{t}=F \mathbf{r}$ be an evolution of the form (3.9), where $F$ contains the Frenet frame in columns and where $\mathbf{r}(x)=\left(r_{i}(x)\right) \in \mathbb{R}^{n}$ is an invariant vector, that is, depending on the Frenet curvatures $\kappa_{i}, s$ and their derivatives with respect to $x$. Then, the evolution induced on the curvatures $\kappa_{i}$ and on the invariant arc length by the evolution of the curve is given by

$$
\begin{aligned}
\left(s^{2}\right)_{t} & =\left[\mathcal{D}_{K} \pi(\mathbf{r})\right]_{1}+\left(s^{2} D+D s^{2}\right) r_{1}, \\
\left(\kappa_{1}\right)_{t} & =\left(-D^{3}+\kappa_{1} D+D \kappa_{1}\right) r_{1}+\left(s^{2} D+D s^{2}\right) r_{2}-\kappa_{2} s^{2} r_{3}, \\
\pi(\kappa)_{t} & =\mathcal{B}\left(\pi\left(s^{-2} \mathcal{D}_{K}(\pi(\mathbf{r}))+K_{2} r_{1} e_{1}^{n-1}\right)\right)+\mathcal{C}\left(s^{2} \pi^{2}(\mathbf{r})\right),
\end{aligned}
$$

where $\mathcal{B}$ and $\mathcal{C}$ are the Euclidean operators given in Lemma 1, $D$ denotes the differentiation operator, and where $\mathcal{D}_{K}$ is the operator $\mathcal{D}_{K}=\left(D+K_{2}\right)^{3}-\left(D+K_{2}\right) \kappa_{1}-$ $\kappa_{1}\left(D+K_{2}\right), K_{2}$ as in (2.9).

Proof. The evolution of the curvatures can be obtained evaluating the structural equation

$$
d \omega+\frac{1}{2}[\omega, \omega]=0
$$

on two fields along the 1-parameter family of curves $u(x, t)$. In particular, consider $\left(u_{1}, \rho_{x}\right) \in T_{(u, \rho)} P$ and $\left(u_{t}, \rho_{t}\right) \in T_{(u, \rho)} P$ to be two vector fields defined along the curve $(u, \rho) \in P$ on the principal bundle associated to the conformal Cartan structure of $S$. Recall that, for a homonegeous space $T(G / H) \approx G \times_{H} \mathfrak{g} / \mathfrak{h} \approx$ $P \times_{H} \mathfrak{g} / \mathfrak{h}$ (see $\left.\underline{\mathrm{Sh}}\right)$. If we evaluate the structural equation on $\left(u_{1}, \rho_{x}\right),\left(u_{t}, \rho_{t}\right)$, 
and having in mind that the action of these vector fields on functions is given by differentiation with respect to $x$ or $t$ along $(u, \rho)$, we get

$$
\frac{d}{d t}\left(\omega\left(u_{1}, \rho_{x}\right)\right)-\frac{d}{d x}\left(\omega\left(u_{t}, \rho_{t}\right)\right)+\left[\left(u_{1}, \rho_{x}\right),\left(u_{t}, \rho_{t}\right)\right]-\left[\omega\left(u_{1}, \rho_{x}\right), \omega\left(u_{t}, \rho_{t}\right)\right]=0,
$$

where the first Lie bracket is the Lie bracket of vector fields and the second is the Lie bracket in $\mathfrak{g}$.

Now, since the parameters $x$ and $t$ are independent, the bracket $\left[\left(u_{1}, \rho_{x}\right),\left(u_{t}, \rho_{t}\right)\right]$ vanishes. Also, since $\omega$ is locally the pull-back of the Maurer-Cartan equation in $O(n+1,1)$, we have that $\omega\left(u_{1}, \rho_{x}\right)=\rho^{-1} \rho_{x}$ and $\omega\left(u_{t}, \rho_{t}\right)=\rho^{-1} \rho_{t}$. If we choose $\rho=\rho_{K}$ to be the Frenet moving frame for $u$, using Theorems 6 and 7 and substituting in (5.6) we obtain

$$
\frac{d}{d t} \hat{K}=\frac{d}{d x} R+[\hat{K}, R]
$$

where $R$ is given as in (3.10) and $\hat{K}$ is as in (3.2). (The reader might want to compare this equation to the Kac-Moody Hamiltonian vector field (4.4).)

We next claim that equation (5.7) completely determines all entries in $R$ in terms of $\mathbf{r}$ and $K$. Indeed, if we substitute (3.10) the right-hand side of (5.7) equals

$$
\left(\begin{array}{ccc}
\epsilon^{\prime}+\xi_{1}-\kappa_{1} r_{1}-s^{2} r_{2} & * & 0 \\
\xi^{\prime}+\epsilon \kappa_{1} e_{1}+\epsilon s^{2} e_{2}+ & A^{\prime}+[K, A]+\kappa_{1}\left(e_{1} \mathbf{r}^{T}-\mathbf{r} e_{1}^{T}\right) & \mathbf{r}^{\prime}+K \mathbf{r}-\epsilon e_{1}-A e_{1} \\
K \xi-\kappa_{1} A e_{1}-s^{2} A e_{2} & s^{2}\left(e_{2} \mathbf{r}^{T}-\mathbf{r} e_{2}^{T}\right)+e_{1} \xi^{T}-\xi e_{1}^{T} & \\
0 & * & *
\end{array}\right) .
$$

If we ask this matrix to belong to $T \mathcal{K}$ we get the relations

$$
A e_{1}=\mathbf{r}^{\prime}+K \mathbf{r}-\epsilon e_{1} .
$$

Since $A \in o(n)$, this implies

$$
\epsilon=r_{1}^{\prime}
$$

Also, if

$$
A=\left(\begin{array}{cc}
0 & \mathbf{a}_{0}^{T} \\
-\mathbf{a}_{0} & A_{2}
\end{array}\right)
$$

then $\mathbf{a}_{0}=-\pi(\mathbf{r})^{\prime}-K_{2} \pi(\mathbf{r})$. The upper left corner in (5.8) also determines $\xi_{1}=$ $-r_{1}^{\prime \prime}+\kappa_{1} r_{1}+s^{2} r_{2}$.

Looking next at the $o(n)$ central block, we can rewrite it as

$$
\left(\begin{array}{c}
0 \\
-\mathbf{a}_{0}^{\prime}-K_{2} \mathbf{a}_{0} \\
-\kappa_{1} \pi(\mathbf{r})-\pi(\xi)+s^{2} r_{1} e_{1}^{n-1}
\end{array} A_{2}^{\prime}+\left[K_{2}, A_{2}\right]+s^{2}\left(\begin{array}{cc}
0 & \pi^{2}(\mathbf{r})^{T} \\
-\pi^{2}(\mathbf{r}) & 0
\end{array}\right)\right) .
$$

Therefore, (5.7) belonging to $T \mathcal{K}$ implies

$$
\pi(\xi)=-\mathbf{a}_{0}^{\prime}-K_{2} \mathbf{a}_{0}-\kappa_{1} \pi(\mathbf{r})+s^{2} r_{1} e_{1}^{n-1}=\left(D+K_{2}\right)^{2} \pi(\mathbf{r})-\kappa_{1} \pi(\mathbf{r})+s^{2} r_{1} e_{1}^{n-1}
$$

and also

$$
A_{2}^{\prime}+\left[K_{2}, A_{2}\right]+s^{2}\left(\begin{array}{cc}
0 & \pi^{2}(\mathbf{r})^{T} \\
-\pi^{2}(\mathbf{r}) & 0
\end{array}\right) \in \mathfrak{g}_{-1}^{n-1} \oplus \mathfrak{g}_{1}^{n-1}
$$


where $\mathfrak{g}_{-1}^{n-1} \oplus \mathfrak{g}_{1}^{n-1}$ are the -1 and +1 components of the gradation of $o(n-1)$. Application of Lemma 1 ensures that $A_{2}=B\left(\pi\left(A_{2} e_{1}^{n-1}\right)\right)$ and the $\mathfrak{g}_{-1}^{n-1}$ component of (5.10) is

$$
\mathcal{B}\left(\pi\left(A_{2} e_{1}^{n-1}\right)\right)+\mathcal{C}\left(s^{2} \pi^{2}(\mathbf{r})\right) .
$$

The evolution of $\pi(\kappa)$ is given by the $\mathfrak{g}_{-1}^{n-1}$ component of (5.10), that is, by (5.11).

Finally, the left column of (5.8) implies

$$
s^{2} \pi^{2}\left(A e_{2}\right)=\pi\left[\left(D+K_{2}\right) \pi(\xi)+\kappa_{1} \mathbf{a}_{0}\right] .
$$

From here

$$
s^{2} \pi\left(A_{2} e_{1}^{n-1}\right)=\pi\left(\left(D+K_{2}\right) \pi(\xi)+\kappa_{1} \mathbf{a}_{0}\right)=\pi\left(\mathcal{D}_{K} \pi(\mathbf{r})+K_{2} s^{2} r_{1} e_{1}^{n-1}\right)
$$

where $\mathcal{D}_{K}=\left(D+K_{2}\right)^{3}-\left(D+K_{2}\right) \kappa_{1}-\kappa_{1}\left(D+K_{2}\right)$. Hence, the evolution of $\pi(\kappa)$ is as given in the statement of the theorem.

The evolution of $\kappa_{1}$ is given by the first entry of the left column in (5.8), namely

$$
\left(\kappa_{1}\right)_{t}=\xi_{1}^{\prime}+\epsilon \kappa_{1}-s^{2}\left(A e_{2}\right)_{1}
$$

where $\left(A e_{2}\right)_{1}$ represents the first entry of $A e_{2}$, that is, the first entry of $\mathbf{a}_{0}=$ $-\left(D+K_{2}\right) \pi(\mathbf{r})$. Since $\xi_{1}=-r_{1}^{\prime \prime}+\kappa_{1} r_{1}+s^{2} r_{2}$ and $\epsilon=r_{1}^{\prime}$, the formula follows.

The evolution of $s^{2}$ is given by the second entry on the left column in (5.8), namely

$$
\left(s^{2}\right)_{t}=\left[\left(D+K_{2}\right) \pi(\xi)\right]_{1}+\epsilon s^{2}+\kappa_{1}\left(\mathbf{a}_{0}\right)_{1} .
$$

Substituting the corresponding values we obtain the equation in the theorem.

The theorem describing the evolution of natural curvatures is given next.

Theorem 14. Let $u_{t}=F \mathbf{r}$ be an evolution of the form (3.9), where $F$ contains the natural frame in columns and where $\mathbf{r}(x)=\left(r_{i}(x)\right) \in \mathbb{R}^{n}$ is an invariant vector, that $i s$, depending on the natural curvatures $\nu_{i}, s$ and their derivatives with respect to the parameter $x$. Then, the evolution induced on the curvatures $\nu_{i}$ by the evolution of the curve is given by

$$
\begin{aligned}
\left(s^{2}\right)_{t} & =\left[\mathcal{D}_{N} \pi(\mathbf{r})\right]_{1}+\left(s^{2} D+D s^{2}\right) r_{1}, \\
\left(\nu_{1}\right)_{t} & =\left(-D^{3}+\nu_{1} D+D \nu_{1}\right) r_{1}+\left(s^{2} D+D s^{2}\right) r_{2}-s^{2} \pi(\nu) \cdot \pi^{2}(\mathbf{r}), \\
\pi(\nu)_{t} & =D\left(s^{-2} \pi\left(\mathcal{D}_{N}(\pi(\mathbf{r}))+r_{1} \pi(\nu)\right)\right) \\
& +D^{-1}\left(\pi(\nu) \mathbf{a}_{\mathbf{1}}{ }^{T}-\mathbf{a}_{\mathbf{1}} \pi(\nu)^{T}\right) \pi(\nu)-s^{2} \pi^{2}(\mathbf{r}),
\end{aligned}
$$

where $D^{-1}$ denotes the formal inverse of the differentiation operator (i.e. the formula makes sense only if $\left(\pi(u) \mathbf{a}_{\mathbf{1}}^{T}-\mathbf{a}_{\mathbf{1}} \pi(u)^{T}\right)$ is a total derivative), and where $\mathbf{a}_{1}=-s^{-2} \pi\left(\mathcal{D}_{N} \pi(\mathbf{r})\right)-r_{1} \pi(\nu)$ and $\mathcal{D}_{N}$ is the operator $\mathcal{D}_{N}=\left(D+N_{2}\right)^{3}-$ $\left(D+N_{2}\right) \kappa_{1}-\kappa_{1}\left(D+N_{2}\right), N_{2}$ as in (2.11).

Proof. We will simply do a direct calculation as done in the previous theorem. We evaluate first the structure equation along the curve $\left(u, \rho_{K}\right)$ on the two fields $\left(u_{1},\left(\rho_{K}\right)_{x}\right)$ and $\left(u_{t},\left(\rho_{K}\right)_{t}\right)$, where $\rho_{K}$ is the moving frame associated to the natural frame as in Theorem 5 As in the previous theorem, we get

$$
\frac{d}{d t} \omega\left(u_{1},\left(\rho_{K}\right)_{x}\right)=\frac{d}{d x} \omega\left(u_{t},\left(\rho_{K}\right)_{t}\right)+\left[\omega\left(u_{1},\left(\rho_{K}\right)_{x}\right), \omega\left(u_{t},\left(\rho_{K}\right)_{t}\right)\right] .
$$


Using Theorems 6 and 7 we conclude that

$$
\frac{d}{d t} \hat{K}=\frac{d}{d x}\left(\begin{array}{ccc}
\epsilon & \mathbf{r}^{T} & 0 \\
\xi & A & \mathbf{r} \\
0 & \xi^{T} & -\epsilon
\end{array}\right)+\left[\hat{K},\left(\begin{array}{ccc}
\epsilon & \mathbf{r}^{T} & 0 \\
\xi & A & \mathbf{r} \\
0 & \xi^{T} & -\epsilon
\end{array}\right)\right]
$$

where $\hat{K}$ is as in Theorem 6 in the natural frame case. The right-hand side of this equation is given by

$$
\left(\begin{array}{ccc}
\epsilon^{\prime}+\xi_{1}-\nu_{1} r_{1}-s^{2} r_{2} & * & 0 \\
\xi^{\prime}+\epsilon \nu_{1} e_{1}+s^{2} \epsilon e_{2}+ & A^{\prime}+[K, A]+\nu_{1}\left(e_{1} \mathbf{r}^{T}-\mathbf{r} e_{1}^{T}\right) & \mathbf{r}^{\prime}+K \mathbf{r}-\epsilon e_{1}-A e_{1} \\
K \xi-\nu_{1} A e_{1}-s^{2} A e_{2} & s^{2}\left(e_{2} \mathbf{r}^{T}-\mathbf{r} e_{2}^{T}\right)+e_{1} \xi^{T}-\xi e_{1}^{T} & \\
0 & * & *
\end{array}\right)
$$

which needs to belong to $T \mathcal{N}$. If $A$ is written as in (5.9), straightforward calculations find the values

$$
\begin{array}{cc}
\epsilon=r_{1}^{\prime}, \quad \mathbf{a}_{0}=-\left(D+N_{2}\right) \pi(\mathbf{r})=\left(\begin{array}{l}
-r_{2}^{\prime}+\pi(\nu) \cdot \pi^{2}(\mathbf{r}) \\
-\pi^{2}(\mathbf{r})^{\prime}-r_{2} \pi(\nu)
\end{array}\right), \\
\xi_{1}=-r_{1}^{\prime \prime}+\nu_{1} r_{1}+s^{2} r_{2}, \quad \pi(\xi)=\left(D+N_{2}\right)^{2} \pi(\mathbf{r})-\nu_{1} \pi(\mathbf{r})+s^{2} r_{1} e_{1}^{n-1},
\end{array}
$$

and also if

$$
A_{2}=\left(\begin{array}{cc}
0 & \mathbf{a}_{1}^{T} \\
-\mathbf{a}_{1} & A_{3}
\end{array}\right)
$$

then

$$
\mathbf{a}_{1}=-s^{-2} \pi\left(\mathcal{D}_{N} \pi(\mathbf{r})\right)-r_{1} \pi(\nu),
$$

$\mathcal{D}_{N}$ as in the statement of the theorem. We can also write the central $o(n-1)$ block in (5.14) as

$$
\left(\begin{array}{cc}
0 & * \\
-\mathbf{a}_{1}^{\prime}-A_{3} \pi(\nu)-s^{2} \pi^{2}(\mathbf{r}) & A_{3}^{\prime}-\mathbf{a}_{1} \pi(\nu)^{T}+\pi(\nu) \mathbf{a}_{1}^{T}
\end{array}\right) .
$$

Assuming (5.14) is in $T \mathcal{N}$, we obtain formally

$$
A_{3}=D^{-1}\left(\mathbf{a}_{1} \pi(\nu)^{T}-\pi(\nu) \mathbf{a}_{1}^{T}\right)
$$

and $\pi(\nu)_{t}=-\mathbf{a}_{1}^{\prime}-A_{3} \pi(\nu)-s^{2} \pi^{2}(\mathbf{r})$. Substituting all values obtained we get the evolution for $\pi(\nu)$ stated in the theorem. As before

$\left(\nu_{1}\right)_{t}=\xi_{1}^{\prime}+\epsilon \nu_{1}-s^{2}\left(a_{0}\right)_{1}=\left(-D^{3}+D \nu_{1}+\nu_{1} D\right) r_{1}+\left(s^{2} D+D s^{2}\right) r_{2}-s^{2} \pi(\nu) \cdot \pi^{2}(\mathbf{r})$

and also

$$
\left(s^{2}\right)_{t}=\left[\mathcal{D}_{N} \pi(\mathbf{r})\right]_{1}+\left(s^{2} D+D s^{2}\right) r_{1} .
$$

The final two theorems of this section describe the condition for an evolution of the type (3.9) to induce a Hamiltonian evolution on its curvatures. 
Theorem 15. Let $u_{t}=F \mathbf{r}$ be an evolution of the form (3.9), where $F$ contains the Frenet frame in columns and where $\mathbf{r}(x)=\left(r_{i}(x)\right) \in \mathbb{R}^{n}$ is an invariant vector. Then, if there exists a Hamiltonian functional $h: \mathcal{K} \rightarrow \mathbb{R}$ such that

$$
\left(\begin{array}{c}
h_{1} \\
h_{s} \\
-s^{2} \mathcal{A}(\pi(\mathbf{h}))
\end{array}\right)=\mathbf{r}
$$

where $h_{i}=\frac{\delta h}{\delta \kappa_{i}}, i=1, \ldots, n-1, h_{s}=\frac{\delta h}{\delta s^{2}}$ and where $\mathbf{h}=\left(h_{i}\right)$, the evolution induced on $\kappa$ by the $u$-evolution is Hamiltonian with respect to the $\mathcal{K}$-reduced bracket, with Hamiltonian functional $h$. If an evolution $u_{t}=F \hat{\mathbf{r}}$ induces a $\kappa$-evolution which is Hamiltonian with respect to a functional $h$, then the evolution $u_{t}=F \mathbf{r}$, with $\mathbf{r}$ as in (5.16), induces the same $\kappa$-evolution as $u_{t}=F \hat{\mathbf{r}}$.

Proof. We will first deal with a rewritting of the reduced bracket. Comparison of an explicit expression for the reduced bracket with evolution (5.5) will automatically yield the result of the theorem.

From the description of the reduced bracket (4.14), if $h$ and $g$ are functionals on $\mathcal{K}$, their brackets are defined by

$$
\{h, g\}_{R}(K)=\int_{S^{1}} \operatorname{tr}\left(\left(H^{\prime}+[K, H]\right) G\right) d x
$$

where $H$ and $G$ are the variational derivatives of extensions of $h$ and $g$ to $L o(n+1,1)^{*}$, respectively, with the additional condition

$$
H^{\prime}+[\hat{K}, H] \in \mathfrak{h}^{0}, \quad G^{\prime}+[\hat{K}, G] \in \mathfrak{h}^{0}
$$

along $\mathcal{K}$, where $\mathfrak{h}$ is the algebra associated to (4.12) and where $\mathfrak{h}^{0}$ denotes elements in $\mathfrak{g}^{*}$ vanishing on $\mathfrak{h}$, that is,

$$
h^{0}=\left\{\left(\begin{array}{cccc}
0 & & 0 & 0 \\
* & \left(\begin{array}{ccc}
0 & * \\
* & 0_{n-1 \times n-1}
\end{array}\right) & 0 \\
0 & & * & 0
\end{array}\right)\right\} .
$$

Both $H$ and $G$ will have the form

$$
\left(\begin{array}{ccc}
b & \mathbf{n}^{T} & 0 \\
\mathbf{m} & B & \mathbf{n} \\
0 & \mathbf{m}^{T} & -b
\end{array}\right)
$$

and, because they are variational derivatives of extensions of $h$ and $g$, if $h_{i}=\frac{\delta h}{\delta \kappa_{i}}$, the +1 gradation component of the $B$-block in $H$ will be given by the vector $\left(0, h_{2}, \ldots, h_{n-1}\right)$ and its negative. For the same reason, if $\mathbf{n}=\left(n_{i}\right)$ in $H$, the first entry of $\mathbf{n}$ is given by $h_{1}$, that is, $n_{1}=h_{1}$, and the second is $n_{2}=h_{s}=\frac{\delta h}{\delta s^{2}}$. The same situation holds for $g$.

Next, if $\mathbf{m}=\left(m_{i}\right)$ and we write out (5.17), for either $h$ or $g$ we get

$$
\left(\begin{array}{ccc}
b^{\prime}-\kappa_{1} n_{1}-s^{2} n_{2}+m_{1} & \left(\mathbf{n}^{\prime}+K \mathbf{n}-b e_{1}-B e_{1}\right)^{T} & 0 \\
\mathbf{m}^{\prime}+\kappa_{1} b e_{1}+s^{2} b e_{2} & B^{\prime}+[K, B]+s^{2}\left(e_{2} \mathbf{n}^{T}-\mathbf{n} e_{2}^{T}\right) & \\
+K \mathbf{m}-\kappa_{1} B e_{1}-s^{2} B e_{2} & +\kappa_{1}\left(e_{1} \mathbf{n}^{T}-\mathbf{n} e_{1}^{T}\right)+e_{1} \mathbf{m}^{T}-\mathbf{m} e_{1}^{T} & * \\
0 & * & *
\end{array}\right)
$$


so that condition (5.17) implies $b=h_{1}^{\prime}$ and $m_{1}=s^{2} h_{s}-h_{1}^{\prime \prime}+\kappa_{1} h_{1}$. If

$$
B=\left(\begin{array}{cc}
0 & \mathbf{b}_{0}^{T} \\
-\mathbf{b}_{0} & B_{2}
\end{array}\right),
$$

we also get $\mathbf{b}_{0}=-\pi(\mathbf{n})^{\prime}-K_{2} \pi(\mathbf{n})$.

The central $o(n)$ block becomes

$$
\begin{gathered}
\left(\begin{array}{cc}
0 & * \\
-\mathbf{b}_{0}^{\prime}-K_{2} \mathbf{b}_{0}-\kappa_{1} \pi(\mathbf{n})-\pi(\mathbf{m}) & B_{2}^{\prime}+\left[K_{2}, B_{2}\right]
\end{array}\right) \\
+s^{2}\left(\begin{array}{ccc}
0 & -n_{1} & 0 \\
n_{1} & 0 & \pi^{2}(\mathbf{n})^{T} \\
0 & -\pi^{2}(\mathbf{n}) & 0
\end{array}\right),
\end{gathered}
$$

which implies

$$
B_{2}^{\prime}+\left[K_{2}, B_{2}\right]+s^{2}\left(\begin{array}{cc}
0 & \pi^{2}(\mathbf{n})^{T} \\
-\pi^{2}(\mathbf{n}) & 0
\end{array}\right)=0 .
$$

Given that the +1 gradation component of $B_{2}$ as an element of $g l(n-1)$ is given by the vector $\pi(\mathbf{h})=\left(h_{2}, \ldots, h_{n-1}\right)$, application of Lemma 2 implies that $B_{2}=$ $A(\pi(\mathbf{h}))$ and

$$
B_{2}^{\prime}+\left[K_{2}, B_{2}\right]=\left(\begin{array}{cc}
0 & (\mathcal{A} \pi(\mathbf{h}))^{T} \\
-\mathcal{A} \pi(\mathbf{h}) & 0
\end{array}\right) .
$$

Therefore, $\pi^{2}(\mathbf{n})=-s^{-2} \mathcal{A} \pi(\mathbf{h})$ and

$$
\mathbf{n}_{h}=\left(\begin{array}{c}
h_{1} \\
h_{s} \\
-s^{-2} \mathcal{A} \pi(\mathbf{h})
\end{array}\right) .
$$

We can now use all of this information to write an explicit formula for the reduced Poisson bracket. If $h, g: \mathcal{K} \rightarrow \mathbb{R}$ are two functionals and $\mathcal{H}, \mathcal{G}: \mathcal{M} \rightarrow \mathbb{R}$ are two extensions to $L o(n+1,1)^{*}$ with variational derivatives $H$ and $G$ of the form (5.18), the entries determined as above, the Poisson bracket of $h$ and $g$ is given by

$$
\{h, g\}_{R}(\hat{K})=\int_{S^{1}} \operatorname{tr}\left(\left(H^{\prime}+[\hat{K}, H]\right) G\right) d x
$$

where

$$
H=\left(\begin{array}{ccc}
b_{h} & \mathbf{n}_{h}^{T} & 0 \\
\mathbf{m}_{h} & B_{h} & \mathbf{n}_{h} \\
0 & \mathbf{m}_{h}^{T} & -b_{h}
\end{array}\right), \quad G=\left(\begin{array}{ccc}
b_{g} & \mathbf{n}_{g}^{T} & 0 \\
\mathbf{m}_{g} & B_{g} & \mathbf{n}_{g} \\
0 & \mathbf{m}_{g}^{T} & -b_{g}
\end{array}\right) .
$$

Since $H^{\prime}+[\hat{K}, H] \in \mathfrak{h}^{0}$, we can rewrite this bracket as

$$
\begin{aligned}
& \{h, g\}_{R}(\hat{K})=\int_{S^{1}}\left(b_{0}\right)_{g} \cdot\left(\left(D+K_{2}\right)^{2} \pi\left(\mathbf{n}_{h}\right)-\kappa_{1} \pi\left(\mathbf{n}_{h}\right)+s^{2}\left(n_{1}\right)_{h} e_{1}^{n-1}-\pi\left(\mathbf{m}_{h}\right)\right) d x \\
& +\int_{S^{1}} \mathbf{n}_{g} \cdot\left(\begin{array}{c}
\left(m_{1}\right)_{h}^{\prime}+\kappa_{1} h_{1}^{\prime}+s^{2}\left[\left(D+K_{2}\right) \pi\left(\mathbf{n}_{h}\right)\right]_{1} \\
\left.\left(D+K_{2}\right) \pi\left(\mathbf{m}_{h}\right)-\kappa_{1}\left(D+K_{2}\right) \pi\left(\mathbf{n}_{h}\right)+s^{2} h_{1}^{\prime} e_{1}^{n-1}-s^{2}\left(B_{2}\right)_{h} e_{1}^{n-1}\right) d x
\end{array}\right.
\end{aligned}
$$


Using that $\left(B_{2}\right)_{h} e_{1}^{n-1}=\left(\begin{array}{c}0 \\ -\mathcal{R} \pi(\mathbf{h})\end{array}\right)$ and Proposition 2 the above becomes

$$
\begin{aligned}
& \{f, g\}_{R}(\hat{K})=\int_{S^{1}} g_{1}\left(\left(m_{1}\right)_{h}^{\prime}+\kappa_{1} h_{1}^{\prime}+s^{2}\left[\left(D+K_{2}\right) \pi\left(\mathbf{n}_{h}\right)\right]_{1}\right) d x \\
& +\int_{S^{1}} \pi\left(\mathbf{n}_{g}\right) \cdot\left(\mathcal{D}_{K} \pi\left(\mathbf{n}_{h}\right)+\left(D s^{2}+s^{2} D+K_{2}\right) h_{1} e_{1}^{n-1}+\left(\begin{array}{c}
0 \\
s^{2} \mathcal{C}^{*} \pi(\mathbf{h})
\end{array}\right)\right) d x
\end{aligned}
$$

where $[,]_{1}$ represents the first entry of the vector. Notice that $\pi\left(\mathbf{m}_{h}\right)$ and $\pi\left(\mathbf{m}_{g}\right)$ are not involved. This simply reflects the fact that the reduced bracket is independent of the extensions of the operators $h$ and $g$ as far as their variational derivatives vanish on the subbundle E. Using (5.22) and Proposition 2 we finally get

$$
\begin{aligned}
& \{h, g\}_{R}(\kappa)= \\
& \int_{S^{1}} g_{1}\left(\left(-D^{3}+\kappa_{1} D+D \kappa_{1}\right) h_{1}+\left(s^{2} D+D s^{2}\right) h_{s}+s^{2}\left[K_{2} \pi\left(\mathbf{n}_{h}\right)\right]_{1}\right) d x \\
+ & \int_{S^{1}} g_{s}\left(\left[\mathcal{D}_{K} \pi\left(\mathbf{n}_{h}\right)\right]_{1}+\left(s^{2} D+D s^{2}\right) h_{1}\right) d x \\
+ & \int_{S^{1}} \pi(\mathbf{g}) \cdot\left(\mathcal{B}\left(\pi\left(s^{-2} \mathcal{D}_{K} \pi\left(\mathbf{n}_{h}\right)+K_{2} h_{1} e_{1}^{n-1}\right)\right)+\mathcal{B C}^{*} \pi(\mathbf{h})\right) d x,
\end{aligned}
$$

with the right-hand side in each term describing the reduced Hamiltonian vector fields associated to the functional $h$. Finally, notice that, from Proposition 2 we have

$$
B \mathcal{C}^{*} \pi(\mathbf{h})=-\mathcal{C} \mathcal{A} \pi(\mathbf{h})=\mathcal{C}\left(s^{2} \pi^{2}\left(\mathbf{n}_{h}\right)\right) .
$$

Therefore, if $\mathbf{r}=\mathbf{n}_{h}$, the projection on the last $n-2$ components of both reduced Hamiltonian evolution and evolution of the conformal curvatures coincide. The first two components of the reduced Hamiltonian evolution also coincide with the invariant evolution of $\kappa_{1}$ and $s^{2}$, and so the theorem is proved.

Assume we are now working formally, not in the periodic case.

Theorem 16. Let $u_{t}=F \mathbf{r}$ be an evolution of the form (3.9), where $F$ contains the natural frame in columns and where $\mathbf{r}(x)=\left(r_{i}(x)\right) \in \mathbb{R}^{n}$ is an invariant vector. Then, if there exists a Hamiltonian functional $h: \mathcal{N} \rightarrow \mathbb{R}$ such that

$$
\left(\begin{array}{c}
h_{1} \\
h_{s} \\
-D(\pi(\mathbf{h}))-D^{-1}\left(\pi(\mathbf{h}) \pi(\nu)^{\mathbf{T}}-\pi(\nu) \pi(\mathbf{h})^{\mathbf{T}}\right) \pi(\nu)
\end{array}\right)=\mathbf{r}
$$

where $h_{i}=\frac{\delta h}{\delta \nu_{i}}, i=1, \ldots, n-1, h_{s}=\frac{\delta h}{\delta s^{2}}$ and where $\mathbf{h}=\left(h_{i}\right)$, the evolution induced on $\nu$ by the $u$-evolution is Hamiltonian with respect to the $\mathcal{N}$-reduced bracket, with Hamiltonian functional $h$.

If an evolution $u_{t}=F \hat{\mathbf{r}}$ induces a $\nu$-evolution which is Hamiltonian with respect to a functional $h$, then the evolution $u_{t}=F \mathbf{r}$, with $\mathbf{r}$ as in (5.24), induces the same $\nu$-evolution as $u_{t}=F \hat{\mathbf{r}}$.

Proof. Again, one proceeds as in the previous theorem. We need to write explicitly the reduced bracket and compare it to evolution (5.13). The choice (5.24) will make both evolutions identical, and the theorem will follow. 
Again, given two functionals $h, g: \mathcal{N} \rightarrow \mathbb{R}$, we choose two extensions to $\operatorname{Lo}(n+1,1)^{*}$ such that their Hamiltonian vector fields vanish on $\mathfrak{h}$, that is,

$$
H^{\prime}+[\hat{K}, H] \in \mathfrak{h}^{0}, \quad G^{\prime}+[\hat{K}, G] \in \mathfrak{h}^{0}
$$

where $\mathfrak{h}$ is the algebra associated to (4.12), and where $H$ and $G$ are the variational derivatives of the extensions of $h$ and $g$ respectively. Here $\hat{K}$ is as in (3.2) for the natural case. Since we are extending to $L o(n+1,1)^{*}, H$ and $G$ will be also of the form

$$
B=\left(\begin{array}{ccc}
b & \mathbf{n}^{T} & 0 \\
\mathbf{m} & B & \mathbf{n} \\
0 & \mathbf{m}^{T} & -b
\end{array}\right)
$$

If $\mathbf{n}=\left(n_{i}\right), \mathbf{m}=\left(m_{i}\right)$ and $B=\left(\begin{array}{cc}0 & \mathbf{b}_{0}^{T} \\ -\mathbf{b}_{0} & B_{2}\end{array}\right)$, one can find directly that (5.25) determines the values

$$
\begin{array}{ccc}
n_{1}=h_{1}, & n_{2}=h_{s}, & m_{1}=-h_{1}^{\prime \prime}+\nu_{1} h_{1}+s^{2} h_{s}, \\
b=h_{1}^{\prime}, & \mathbf{b}_{0}=-\left(D+N_{2}\right) \pi(\mathbf{n})=-\pi(\mathbf{n})^{\prime}-\left(\begin{array}{c}
-\pi(\nu) \cdot \pi^{2}(\mathbf{n}) \\
n_{2} \pi(\nu)
\end{array}\right),
\end{array}
$$

where $N_{2}$ is given as in (2.11). The study of the central block in (5.19), with the natural data, results in

$$
B_{2}^{\prime}+\left[N_{2}, B_{2}\right]+s^{2}\left(\begin{array}{cc}
0 & \pi^{2}(\mathbf{n})^{T} \\
-\pi^{2}(\mathbf{n}) & 0
\end{array}\right)=0 .
$$

If $B_{2}=\left(\begin{array}{cc}0 & \mathbf{b}_{1}^{T} \\ -\mathbf{b}_{1} & B_{3}\end{array}\right)$, automatically one has that $\mathbf{b}_{1}=\pi(\mathbf{h})$, since the matrix (5.26) represents the variational derivative of an extension of a functional $h$ defined on $\mathcal{N}$. Besides, (5.28) implies

$$
B_{3}=D^{-1}\left(\pi(\mathbf{h}) \pi(\nu)^{T}-\pi(\nu) \pi(\mathbf{h})^{T}\right),
$$

and

$$
\pi^{2}(\mathbf{n})=-s^{-2}\left[\pi(\mathbf{h})^{\prime}+D^{-1}\left(\pi(\mathbf{h}) \pi(\nu)^{T}-\pi(\nu) \pi(\mathbf{h})^{T}\right) \pi(\nu)\right] .
$$

With this information, if $H$ and $G$ are given as in (5.23), the reduced bracket is given by

$$
\begin{aligned}
& \{h, g\}_{N R}(\nu)=\int \operatorname{tr}\left(\left(H^{\prime}+[\hat{K}, H]\right) G\right) d x \\
& \quad=\int \mathbf{n}_{g} \cdot\left(\begin{array}{c}
m_{1}^{\prime}+\nu_{1} h_{1}^{\prime}+s^{2} h_{s}^{\prime}-s^{2} \pi(\nu) \cdot \pi^{2}\left(\mathbf{n}_{h}\right) \\
s^{2} h_{1}^{\prime} e_{1}^{n-1}-\nu_{1}\left(D+N_{2}\right) \pi\left(\mathbf{n}_{h}\right)+s^{2}\left(\begin{array}{c}
0 \\
\pi(\mathbf{h})
\end{array}\right)+\left(D+N_{2}\right) \pi\left(\mathbf{m}_{h}\right)
\end{array}\right) d x \\
& \quad+\int-\left(\left(D+N_{2}\right) \pi\left(\mathbf{n}_{g}\right)\right) \cdot\left(\left(D+N_{2}\right)^{2} \pi\left(\mathbf{n}_{h}\right)-\nu_{1} \pi\left(\mathbf{n}_{h}\right)+s^{2} h_{1} e_{1}^{n-1}-\pi\left(\mathbf{m}_{h}\right)\right) d x .
\end{aligned}
$$


Further computations yield the formula

$$
\begin{aligned}
\{h, g\}_{N R}(\nu) & =\int g_{1}\left(\left(-D^{3}+D \nu_{1}+\nu_{1} D\right) h_{1}\right. \\
& \left.+\left(s^{2} D+D s^{2}\right) h_{s}-s^{2} \pi(\nu) \cdot \pi^{2}\left(\mathbf{n}_{h}\right)\right) d x \\
+ & \int g_{s} \cdot\left(\left[\mathcal{D}_{N} \pi\left(\mathbf{n}_{h}\right)\right]_{1}+\left(s^{2} D+D s^{2}\right) h_{1}\right) d x \\
+ & \int \pi^{2}\left(\mathbf{n}_{g}\right) \cdot\left(\left(\pi\left(\mathcal{D}_{N} \pi\left(\mathbf{n}_{h}\right)\right)+s^{2} h_{1} \pi(\nu)+s^{2} \pi(\mathbf{h})\right) d x .\right.
\end{aligned}
$$

If we assume $\mathbf{r}=\mathbf{n}_{h}$, then the companion factor to $g_{1}$ and $g_{s}$ above coincide with the evolution of $\nu_{1}$ and $s^{2}$ in the invariant evolution of the curvatures (5.13). Finally we only need to rewrite the $\pi^{2}\left(\mathbf{n}_{f}\right)$ companion in (5.29) and decide which factor this part will induce on $\pi(\mathbf{g})$. For this, we use the relation

$$
\begin{aligned}
\int[ & \left.D^{-1}\left(\pi(\mathbf{g}) \pi(\nu)^{T}-\pi(\nu) \pi(\mathbf{g})^{T}\right) \pi(\nu)\right] \cdot X d x \\
& =\int \pi(\mathbf{g}) \cdot D^{-1}\left(\pi(\nu) X^{T}-X \pi(\nu)^{T}\right) \pi(\nu) d x
\end{aligned}
$$

and substitute $X=Y+\pi(\mathbf{h})$ where

$$
Y=s^{-2} \pi\left(\mathcal{D}_{N}\left(\pi\left(\mathbf{n}_{h}\right)\right)+h_{1} \pi(\nu)\right)
$$

(the factor accompanying $\pi^{2}\left(\mathbf{n}_{g}\right)$ is $s^{2}(Y+\pi(\mathbf{h}))$ ). After some calculations we obtain that the factor that multiplies $\pi(\mathbf{g})$ in (5.29) equals

$$
D^{-1}\left(Y \pi(\nu)^{T}-\pi(\nu) Y\right) \pi(\nu)-s^{2} \pi^{2}\left(\mathbf{n}_{h}\right)+D\left(s^{-2} \pi\left(\mathcal{D}_{N} \pi\left(\mathbf{n}_{h}\right)\right)\right)+D h_{1} \pi(\nu) .
$$

If $\mathbf{r}=\mathbf{n}_{h}$, then $Y=-\mathbf{a}_{1}$, where $\mathbf{a}_{1}$ is as in (5.15). This implies that the projection of the reduced Hamiltonian vector field associated to $h$ on $\mathbb{R}^{n-2}$ is identical to the evolution induced on $\pi(\nu)$ as in (5.13). The theorem follows.

Example 2. Consider the functional $h: \mathcal{N} \rightarrow \mathbb{R}$,

$$
h(\nu, s)=\int\left(\frac{1}{2}\left\|\pi(\nu)^{\prime}\right\|^{2}+g\left(\nu_{1}, s\right)\right) d x .
$$

This choice gives the values $h_{i}=-\nu_{i}^{\prime \prime}$ for $i=2, \ldots, n-1$ and arbitrary values for $h_{1}=\frac{\delta g}{\delta \nu_{1}}$ and $h_{s}=\frac{\delta g}{\delta s^{2}}$. Therefore, relation (5.24) gives the vector

$$
\mathbf{r}=\left(\begin{array}{c}
h_{1} \\
h_{s} \\
\pi(\nu)^{\prime \prime \prime}+\pi(\nu)^{\prime}\|\pi(\nu)\|^{2}-\frac{1}{2} \pi(\nu)\left(\|\pi(\nu)\|^{2}\right)^{\prime}
\end{array}\right) .
$$

Evolution $u_{t}=F \mathbf{r}$, with $\mathbf{r}$ as above and any choice of $g\left(\nu_{1}, s\right)$, will be Hamiltonian with respect to the reduced Poisson bracket.

Borrowing ideas from the Euclidean case, the choice

$$
h(\nu)=\int\left(\frac{1}{2}\left(\left\|\pi(\nu)^{\prime}\right\|^{2}-\|\pi(\nu)\|^{4}\right)+g\left(\nu_{1}, s\right)\right) d x
$$

produces the values

$$
\mathbf{r}=\left(\begin{array}{c}
h_{1} \\
h_{s} \\
\pi(\nu)^{\prime \prime \prime}+\frac{3}{2} \pi(\nu)^{\prime}\|\pi(\nu)\|^{2}
\end{array}\right)
$$


and again the evolution $u_{t}=F \mathbf{r}$ will induce a Hamiltonian evolution on $s^{2}, \nu$ for any choice of $g\left(\nu_{1}, s\right)$.

\section{Further Questions}

The majority of completely integrable PDEs are integrated with the use of two compatible Poisson structures. If one of the structures is invertible, then a recursion operator can be found to generate an infinite family of preserved quantities of the flow. The system is thus completely integrable in this sense.

The study of integrable systems which appear as evolutions induced on conformal differential invariants by conformally invariant flows is largely unexplored. In this paper we have presented one of the Hamiltonian structures that can be considered for integration. But, the Kac-Moody bracket (4.3) we reduced to find the conformal bracket is known to be compatible with any of the simpler Hamiltonian structures

$$
\{\mathcal{F}, \mathcal{G}\}_{0}(L)=-\int \operatorname{tr}\left([F, G] H_{0}\right) d x
$$

for any constant matrix $H_{0} \in o(n+1,1)^{*}$. Therefore, if any such structures reduce to our submanifolds $\mathcal{K}$ and $\mathcal{N} / O(n-2)$, the resulting bracket will also be compatible with $\{,\}_{R}$ and $\{,\}_{N R}$. The subbundle $E$ used in Theorem 11 leaves this bracket invariant trivially since the right-hand side of (6.1) does not involve the point $L$ explicitly. Therefore, in order for $\left(\operatorname{Lo}(n+1,1)^{*}, \mathcal{M},\{,\}_{0}, E\right)$ to be Poisson reducible, we need condition (4.15) to be satisfied.

As we saw before, $T \mathcal{M}+E=T \mathcal{M}$ and so one would need to make choices of $H_{0}$ with the property

$$
\left[F, H_{0}\right] \in T \mathcal{M}
$$

for any $F=\frac{\delta \mathcal{F}}{\delta M}(M)$ with $F^{\prime}+[M, F] \in \mathfrak{h}^{0}$. An easy choice would be, for example, any $H_{0}$ of the form

$$
\left(\begin{array}{lll}
0 & 0 & 0 \\
* & 0 & 0 \\
0 & * & 0
\end{array}\right)
$$

since the adjoint action of this subalgebra takes $o(n+1,1)$ into $T \mathcal{M}$. In particular, one could explore evolutions that will be connected by a Hasimoto-type transformation, and perhaps evolutions which will be analogous to well-known integrable PDEs appearing in the Euclidean case, notably the Vortex filament flow and vector modified KdV evolutions. Furthermore, in the 3-dimensional Riemannian case of manifolds with constant curvature, the curvature tensor plays an important role on the integration of some of the generalizations of these well-known systems. It would be very interesting to deeply understand this role from a geometrical point of view and to generalise it to other geometries and dimensions.

From the geometrical point of view perhaps a more interesting problem is the study of these Poisson brackets in the general case of a manifold with a $G$-structure. Research in that direction is already under way.

\section{REFERENCES}

[B] R. Bishop. There is more than one way to frame a curve, American Mathematical Monthly, p. 246, 1975. MR51:6604

[C] Cartan, E. La Méthode du Repère Mobile, la Théorie des Groupes Continus et les Espaces Généralisés", Exposés de Géométrie 5, Hermann, Paris, 1935.

[Da] G. Darboux. Leçons sur la Géometrie des espaces de Riemann, Gauthier-Villars, 1910. 
[DSa] A. Doliwa and P. M. Santini. An elementary geometric characterization of the integrable motions of a curve, Phys. Lett. A, 185(4):373-384, 1994. MR95b:58075

[DS] V. G. Drinfel'd and V. V. Sokolov. Lie algebras and equations of Korteweg-de Vries type, In Current problems in mathematics, Vol. 24, Itogi Nauki i Tekhniki, pp. 81-180. Akad. Nauk SSSR Vsesoyuz. Inst. Nauchn. i Tekhn. Inform., Moscow, 1984. MR86h:58071

[F] A. Fialkow. The Conformal Theory of Curves, Transactions of the AMS, 51, pp. 435-568, 1942. MR 3:307e

[FO1] M. Fels, P.J. Olver. Moving coframes. I. A practical algorithm, Acta Appl. Math., pp. 99-136, 1997. MR99c:53012

[FO2] M. Fels, P.J. Olver. Moving coframes. II. Regularization and theoretical foundations. Acta Appl. Math., pp. 127-208, 1999. Mr2000h:58024

[GS] V. Guillemin, S. Stenberg. Variations on a theme by Kepler, Colloquium Publ. AMS, 42, Providence, R.I. 1990. MR93a:58071

[Ha] R. Hasimoto. A soliton on a vortex filament, J. Fluid Mechanics, 51:477-485, 1972.

[Ki] A.A. Kirillov. Infinite dimensional groups: their representation, orbits, invariants, Proc. of International Congress of Math, Helsinki, 1978. MR82a:22022

[K] S. Kobayashi. Transformation Groups in Differential Geometry, Classics in Mathematics, Springer-Verlag, New York, 1972. MR50:8360

[LP1] J. Langer and R. Perline. Poisson geometry of the filament equation, J. Nonlinear Sci., 1(1):71-93, 1991. MR92k:58118

[LP2] J. Langer and R. Perline. Geometric Realizations of Fordy-Kulish Nonlinear Schrödinger Systems, Pacific Journal of Mathematics, 195-1, pp. 157-177, 2000. MR2001i:37114

[M1] G. Marí Beffa. Poisson brackets associated to invariant evolutions of Riemannian curves, to appear in the Pacific Journal of Mathematics, 2004.

[M2] G. Marí Beffa. The theory of differential invariants and KdV Hamiltonian evolutions, Bull. Soc. Math. France, 127(3):363-391, 1999. MR2001m:37142

[M3] G. Marí Beffa. On Relative and Absolute differential invariants of conformal curves, Journal of Lie Theory,13, pp. 213-245, 2003.

[MSW] G. Marí Beffa, J. Sanders, J.P. Wang. Integrable Systems in Three-Dimensional Riemannian Geometry, J. Nonlinear Sc., pp. 143-167, 2002. MR2003f:37137

[MR] J.E. Marsden, T. Ratiu. Reduction of Poisson Manifolds, Letters in Mathematical Physics, 11:pp. 161-169, 1986. MR87h:58067

[MW] Jerrold Marsden and Alan Weinstein. Coadjoint orbits, vortices, and Clebsch variables for incompressible fluids. Phys. D, 7(1-3):305-323, 1983. Order in chaos (Los Alamos, N.M., 1982). MR85g:58039

[O] P.J. Olver. Equivalence, Invariance and Symmetry, Cambridge University Press, Cambridge, UK, 1995. MR 96i:58005

[Sh] R.W. Sharpe. Differential Geometry: Cartan's Generalization of Klein's Erlangen Program, Graduate Texts in Mathematics, Springer, 1997. MR98m:53033

[TT] C.L. Terng and G. Thorbergsson. Completely integrable curve flows on Adjoint orbits, Results Math. 40(1-4):286-309, 2001. MR2002k:37141

[TU] C.L. Terng and K. Uhlenbeck. Poisson actions and scattering theory for integrable systems, Surveys in Differential Geometry: Integrable Systems, A supplement to J. Differential Geometry, 4, pp. 315-402, 2000. MR2001g:37130

[YS] Yukinori Yasui and Norihito Sasaki. Differential geometry of the vortex filament equation. J. Geom. Phys., 28(1-2):195-207, 1998. MR.99m:58119

Department of Mathematics, University of Wisconsin, Madison, Wisconsin 53706

E-mail address: maribeff@math.wisc.edu 